\title{
A DISTRIBUTIONAL ANALYSIS OF REACTION TIMES IN MENTAL ROTATION
}

\author{
A thesis submitted to \\ the Faculty of Graduate Studies and Research \\ in Partial Fulfillment of the requirements for the degree \\ Masters of Arts
}

by

Joey Theberge

Department of Psychology

Carleton University

May, 2008

C2008 Joey Theberge 


$\begin{array}{ll}\begin{array}{l}\text { Library and } \\ \text { Archives Canada }\end{array} & \begin{array}{l}\text { Bibliothèque et } \\ \text { Archives Canada }\end{array} \\ \begin{array}{l}\text { Published Heritage } \\ \text { Branch }\end{array} & \begin{array}{l}\text { Direction du } \\ \text { Patrimoine de l'édition }\end{array} \\ \begin{array}{l}\text { 395 Wellington Street } \\ \text { Ottawa ON K1A 0N4 } \\ \text { Canada }\end{array} & \begin{array}{l}\text { 395, rue Wellington } \\ \text { Ottawa ON K1A 0N4 } \\ \text { Canada }\end{array}\end{array}$

Your file Votre référence ISBN: 978-0-494-43497-0 Our file Notre référence ISBN: 978-0-494-43497-0

NOTICE:

The author has granted a nonexclusive license allowing Library and Archives Canada to reproduce, publish, archive, preserve, conserve, communicate to the public by telecommunication or on the Internet, loan, distribute and sell theses worldwide, for commercial or noncommercial purposes, in microform, paper, electronic and/or any other formats.

The author retains copyright ownership and moral rights in this thesis. Neither the thesis nor substantial extracts from it may be printed or otherwise reproduced without the author's permission.
AVIS:

L'auteur a accordé une licence non exclusive permettant à la Bibliothèque et Archives Canada de reproduire, publier, archiver, sauvegarder, conserver, transmettre au public par télécommunication ou par l'Internet, prêter, distribuer et vendre des thèses partout dans le monde, à des fins commerciales ou autres, sur support microforme, papier, électronique et/ou autres formats.

L'auteur conserve la propriété du droit d'auteur et des droits moraux qui protège cette thèse. $\mathrm{Ni}$ la thèse ni des extraits substantiels de celle-ci ne doivent être imprimés ou autrement reproduits sans son autorisation.
In compliance with the Canadian Privacy Act some supporting forms may have been removed from this thesis.

While these forms may be included in the document page count, their removal does not represent any loss of content from the thesis.
Conformément à la loi canadienne sur la protection de la vie privée, quelques formulaires secondaires ont été enlevés de cette thèse.

Bien que ces formulaires aient inclus dans la pagination, il n'y aura aucun contenu manquant.

\section{Canada}




\begin{abstract}
Twenty participants performed a mental rotation task whereby the capital letters " $F$ ", "L", and "P", were randomly rotated across 840 test trials from 0 to 330 degrees in 30 -degree increments. The main contribution of the present work is that the distributions of obtained reaction times were analysed by fitting them with the ex-Gaussian distributional model. Results indicate that the increases in mean reaction times with increasing rotation angles were accompanied mainly by shifts of the reaction time distributions as a whole, which is consistent with the use of a mental rotation process that is analogous to actual physical rotation. Moreover, performance differences between groups of slower and faster participants were reflected mainly in the size of the tail on the slow end of the reaction time distributions, which is consistent with the periodic use of other types of slower and more analytical cognitive processes by some of the participants.
\end{abstract}


Table of Contents

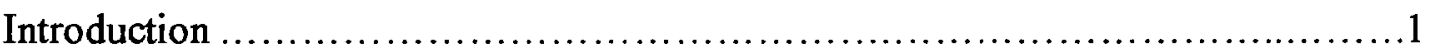

Some Initial Mental Rotation Studies ................................2

Effects of Stimulus Complexity and Practice .............................8

Effects of Stimulus Dimensionality and Type of Task .....................11

Reaction Time Distributions ....................................... 15

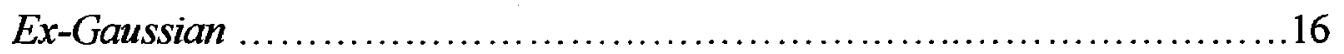

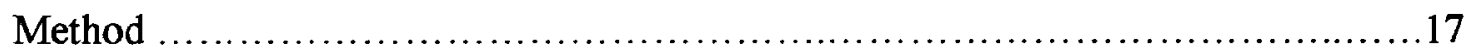

Participants ....................................................... 18

Stimuli and Apparatus .......................................... 18

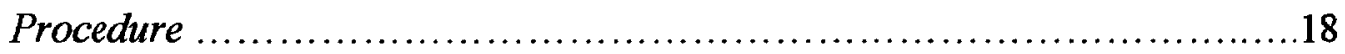

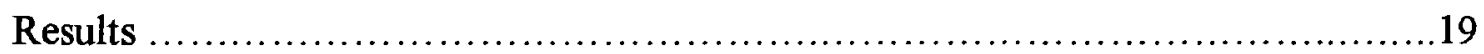

Mean Reaction Time and Accuracy ................................... 19

Mean Reaction Time ................................................20

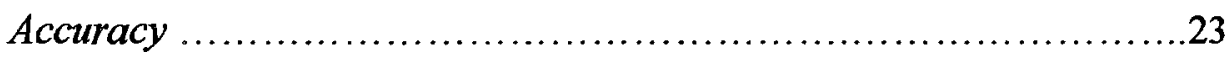

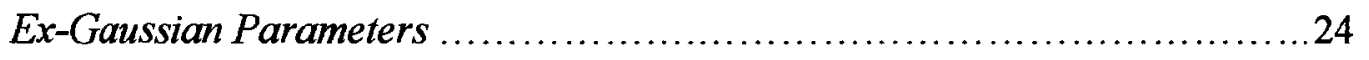

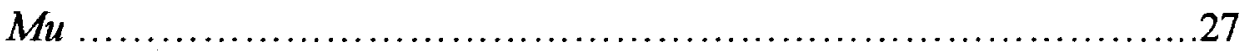

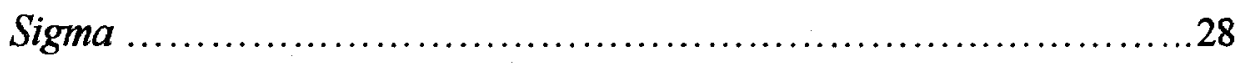

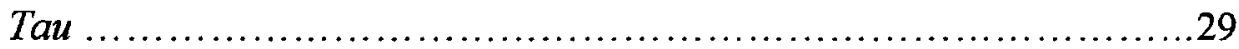

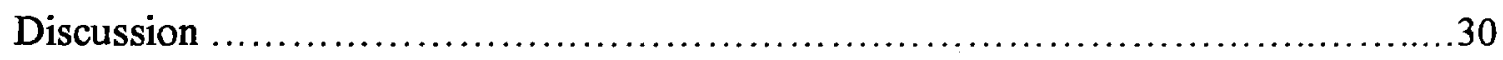

Nonlinearity ......................................................... 32

Standard and Reflected Stimuli ......................................... 34

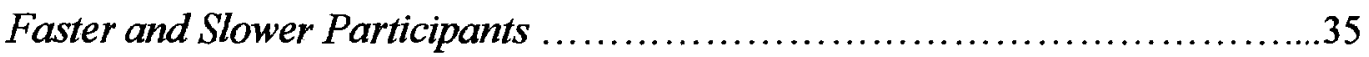




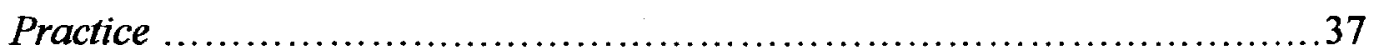

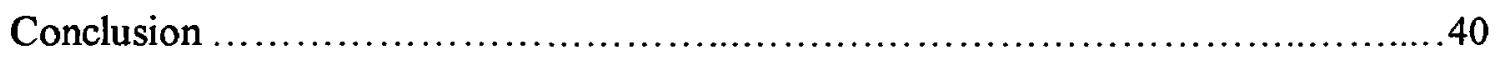

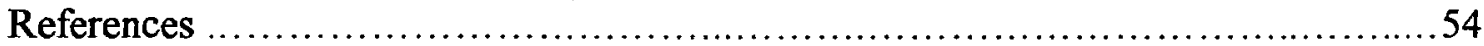


Table 1. Significant Results Found in the ANOVA Analyses for Mean Correct Reaction

Time .42

Table 2. Mean Correct Reaction Time (in ms) as a Function of Angle of Orientation for

Stimulus Type, the First and Last Five Blocks, and the Slowest and Fastest Group of

Participants

Table 3. Proportion Correct as a Function of Angle of Orientation for Stimulus Type and

$\underline{\text { Letters }}$ . .44

Table 4. Significant Results Found in the ANOVA Analyses for $\mathrm{Mu}_{2}$ Sigma, and Tau 45

Table 5. Mu (in ms) as a Function of Angle of Orientation for the First and Last Five

Blocks and the Slowest and Fastest Group of Participants .46

Table 6. Sigma and Tau (in ms) as a Function of Angle of Orientation for the Slowest and

Fastest Group of Participants .47

Table 7. Slope Values for the Mean Reaction Times and the Three Ex-Gaussian

Parameters (in ms/deg) for Responses to Standard Stimuli for both the 0-90 and 90-180

Degree Orientations for the Slowest and Fastest Group of Participants and the First Five and Last Five Blocks of Trials .48

Table A1. Significant Results Found in the ANOVA Analyses for Mean Correct Reaction Time in the Follow-up Study 51

Table A2. Significant Results Found in the ANOVA Analyses for Mu, Sigma, and Tau in the Follow-up Study .52 
Table A3. Slope Values for the Mean Reaction Times and the Three Ex-Gaussian

Parameters (in ms/deg) for Responses to Standard Stimuli for both the 0-90 and 90-180

Degree Orientations for the Slowest and Fastest Group of Participants and the First Five

and Last Five Blocks of Trials in the Follow-up Study ..........................53 


\section{List of Figures}

Figure 1A. Mean correct reaction times and mean values of mu, sigma, and tau for each stimulus type, for each of the first five and the last five blocks of trials at each angle of orientation for the 10 fastest participants

Figure 1B. Mean correct reaction times and mean values of mu, sigma, and tau for each stimulus type, for each of the first five and the last five blocks of trials at each angle of orientation for the 10 slowest participants 58

Figure 2A. Fixed area ( 0.10 per bar), quantile-based, group histograms of the empirical RT distributions of the 10 fastest participants for each of the $0-180$ degree orientations for responses to standard stimuli in the last five blocks of trials. The solid lines represent the ex-Gaussian probability density functions (PDFs) that were the best fit to the corresponding sets of group quantiles values

Figure 2B. Fixed area ( 0.10 per bar), quantile-based, group histograms of the empirical RT distributions of the 10 slowest participants for each of the $0-180$ degree orientations for responses to standard stimuli in the last five blocks of trials. The solid lines represent the ex-Gaussian probability density functions (PDFs) that were the best fit to the corresponding sets of group quantiles values

Figure A1. Mean correct reaction times and mean values of mu, sigma, and tau for each stimulus type, for each of the first five and the last five blocks of trials at each angle of orientation for the 9 fastest participants in the follow-up study

Figure A2. Mean correct reaction times and mean values of mu, sigma, and tau for each stimulus type, for each of the first five and the last five blocks of trials at each angle of

orientation for the 3 slowest participants in the follow-up study ..................64 


\section{List of Appendices}

Appendix 1. The Quantile Maximum Likelihood Estimation (QMLE) Ex-Gaussian

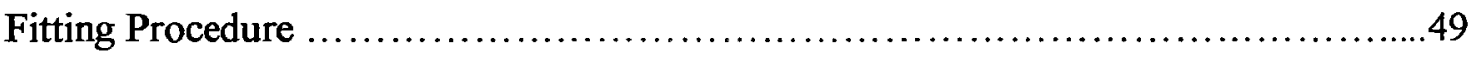

Appendix 2. Results for the Follow-up Study ....................................51 
Introduction

The concept of mental rotation is now 38 -years old. Since its inception, it has been deemed to provide an important window into the nature of spatial representations in the brain and the manner in which such representations can be mentally manipulated. Mental rotation studies typically involve comparative judgments of (i) whether two displayed stimuli are matched in terms of handedness (i.e., whether one has been presented as a mirror image of the other or not), or (b) whether a single displayed stimulus matches the handedness of a stored visual representation regardless of any orientation differences between either the two displayed stimuli or the displayed stimulus and the visual memory representation. Although there is some debate as to the need for mental rotation for other kinds of verification judgments that do not involve handedness (Eley, 1982; Forster, Gebhardt, Lindlar, Seimann, \& Delius, 1996; Raabe, Hoger, \& Delius, 2006), as will be discussed in the following, the results obtained for paradigms involving handedness judgments are very robust and highly suggestive of the fact that participants are mentally rotating one of the displayed stimuli (or the single displayed stimulus) into congruence with the other (or the stored representation).

Much of the research that has been done in the past within this paradigm (much of which will be reviewed in the following) has attempted to delimit the various factors that can affect the speed of mental rotation. Currently, a lot of research interest is focused on the role of the motor processing systems in mental rotation (Raabe et al., 2006; Weidenbauer, Schmid, \& Jansen-Osmann, 2007). Presumably, such research is based on the implicit, but now quite commonly accepted, assumption that mental rotation is indeed a spatial- and imagery-based phenomenon as opposed to one that involves a more 
abstract propositional processing of semantic descriptions (Pylyshyn, 2002). The present study will involve a hitherto unexamined aspect of mental rotation performance, namely, an analysis of the distribution of reaction times obtained for a mental rotation task involving highly familiar capital letter stimuli. Invariably, as for most cognitive paradigms, the analysis of reaction time performance in mental rotation research has focused only on mean reaction times. Like much of the work that will be reviewed, it is hoped that an analysis of the reaction distributions themselves will help to shed more light on exactly what kind of mental processing is involved in the phenomenon known as mental rotation.

\section{Some Initial Mental Rotation Studies}

In the now classic, original mental rotation study by Shepard and Metzler (1971), eight adult participants were tested over 8-10 sessions and were presented with 1600 pairs of perspective line drawings each differing in their relative orientation. Each line drawing consisted of three right angles and ten cubes. Angular departures between the members of each pair varied in increments of 20 degrees. Participants were asked to compare the two stimuli and report whether they were the same or different by judging whether they could be rotated into congruence, where half of the time the shapes of the stimuli were identical and the other half of the time one of the stimuli was displayed as a mirror version of the other. Shepard and Metzler (1971) found that reaction time to report that the two objects had the same shape increased linearly with the angular difference in their portrayed orientation (for both depth and picture plane rotations). On the basis of these findings, and the introspections of their participants, Shepard and Metzler (1971) argued that one of the paired objects is mentally rotated into congruence with the other in 
order to report a shape match or mismatch. Moreover, this process was assumed to be analogous to a physical rotation of a real object.

These initial findings have become the benchmark for further discoveries. Further studies have successfully replicated Shepard and Metzler's work, expanding on it in important ways. In one of these studies, Cooper and Shepard (1973) examined the process of preparation for the presentation of a rotated visual test stimulus. By manipulating the nature and timing of the advance information given to their participants, they examined the time required to make such a preparatory rotation.

Eight participants were tested for six sessions each in Cooper and Shepard's experiment under a number of experimental conditions which varied according to the type and duration of the advance information given. The stimuli were all asymmetrical, alphanumeric letter $(G, J, R)$ or digit $(2,5,7)$ characters which were presented by themselves at testing in either normal or backward (i.e., mirror-image) versions. The rotation of the stimuli varied in six equally spaced orientations around a circle in 60 degree steps. In advance of the rotated test stimulus in four of the conditions, participants were given both identity and orientation information that consisted of a standard stimulus presented in its normal upright position for 2000 ms position followed by an arrow pointing in the direction the test stimulus would appear presented for either 100, 400, 700 , or $1000 \mathrm{~ms}$. In the fifth condition, participants were given both identity and orientation information combined by presenting for $2000 \mathrm{~ms}$ beforehand the standard stimulus already rotated into its test position (followed by a blank screen for $1000 \mathrm{~ms}$ ). In the sixth condition, participants were given no advance information. Participants were 
asked to report manually whether the test stimulus was presented either normally or backwards.

When given no advance information, results supported that of previous work suggesting that participants performed a form of mental rotation in order to manipulate the test stimulus into its upright position so that it could then be compared to a stored mental representation of that stimulus in its normal upright form. It was reported that the slowest reaction times were to stimuli rotated by 180 degrees, and the fact that times fell off symmetrically around 180 degrees indicated that regardless of the clockwise or counterclockwise nature of the rotation, participants performed the same mental rotation in either direction. Cooper and Shepard also observed that the increase in reaction times as the angular departure of the test stimulus from the standard orientation increased was slightly concave as opposed to being strictly linear.

In the four conditions where advance orientation information was given in variable durations, it was reported that increases in the duration of preparatory time that was provided resulted in faster reaction times. At $1000 \mathrm{~ms}$ durations, participants reported having enough time to fully mentally rotate the stimulus into the suggested orientation, which they could then directly compare to the test stimulus. Hence, this condition resulted in similar findings to those obtained in the combined identity and orientation condition, namely, a flat relation between reaction time and angular difference. Conversely, the $100 \mathrm{~ms}$ preparatory duration condition yielded similar findings to those obtained in the no advance information condition in that participants reported that they were often not able to prepare for the orientation of the test stimulus. For the remaining two conditions with the 400 and $700 \mathrm{~ms}$ preparatory durations, reaction 
times still increased with the angular departure of the test stimulus from the upright position but not to the extent as was observed for the shortest preparatory duration. It was concluded that for larger angular departures in these conditions, participants were not able to fully mentally rotate the stimulus during the preparatory period.

Hence, Cooper and Shepard (1973) were able to show that the process of preparation in their study consisted of first generating a mental image of the stimulus and then mentally rotating the image into the pre-designated orientation. If given enough time, participants were able to use their internally generated and pre-rotated internal representation as a template against which to compare the test stimulus. Cooper (1975) then went on to extend this study to include an explicit comparison of pre-stimulus (i.e., preparatory) and post-stimulus mental rotation. Beforehand, though, Cooper examined both the effect of stimulus complexity on mental rotation rate and whether or not the nonlinearity (i.e., the concavity) of the mental rotation reaction time functions for alphanumeric stimuli could be attributed to the familiarity of such stimuli. With respect to the latter issue, Cooper suggested that it was possible that stimuli are more familiar at smaller orientation differences from upright and also that rotation rate can be speeded by familiarity.

Cooper's (1975) first experiment involved eight participants and a stimulus set comprised of eight two-dimensional random polygon forms ranging in complexity as determined by the amount of points on each stimulus (i.e., from 6 points to 24 points). Each participant performed six experimental sessions. The first familiarized the participants with both the standard and reflected versions of the stimuli by presenting them for recognition in their upright position. Sessions 2 through 6 were test sessions 
which consisted, after a short refamiliarization phase, of presenting rotated stimuli by themselves either in their standard or reflected positions. The orientations of the stimuli were equally spaced increments of 60 degrees, ranging from 0 to 300 , and participants responded manually by pushing left- and right-hand buttons.

The mental rotation reaction time functions obtained by Cooper for both standard and reflected responses were "strikingly" linear and parallel across both types of responses. As well, according to Cooper, the fact that reaction times were faster overall for the standard responses supported the notion that participants first compared rotated versions of the test stimulus with a memory representation of the standard, and then required extra time to switch to the reflected response if no initial match was found. Because the forms were learned in a specific orientation, Cooper also argued that the familiarity account for the previously observed non-linearity was not supported (although it was also conceded that alphanumeric stimuli are much more highly overlearned in their upright positions).

A further finding was that mental rotation rates were not significantly related to stimulus complexity. As discussed by Cooper, such a finding is at odds with a propositional account of mental rotation that involves successively manipulating a propositionally based, symbolic visual representation (i.e., a semantic description of the object involving its subparts, its orientation, and the relations between these two aspects) because the complexity of such representations and, hence, the difficulty involved in manipulating them, would almost certainly depend very closely on the actual stimulus complexity. However, Cooper did also acknowledge that a possible alternative explanation for the lack of an effect of stimulus complexity was that participants might 
only have been able to encode the random forms into memory schematically, and perhaps then only used partial patterns or features of each stimulus to make their responses. Such encoding would have eliminated the complexity element of the experiment because if each participant was able to respond using only 4 points, for instance, then a 24-point character would essentially lose its complexity. Finally, Cooper also noted that the possibility that participants might have specifically remembered all of the stimuli in each of their orientations could be ruled out by the fact that the reaction time functions did not become flat by the end of training (although there was some evidence for a slight flattening of these functions across sessions).

Cooper's (1975) second experiment involved the same eight participants and four of the eight previous stimuli. Each participant performed four 1-hour sessions involving trials in which advance identity information regarding the standard form was given for $3000 \mathrm{~ms}$ followed by advance information regarding the orientation of the upcoming test stimulus. Participants were told to push a button when they felt that they were fully prepared for the test stimulus (i.e., when they had fully mentally rotated the standard form into the specified orientation). At that point, the test stimulus appeared and either a standard or reflected vocal response (i.e., "S" or "R", respectively) was required.

Cooper found that the average preparation time was a remarkably linear function of the angular departure of the test stimulus from the trained orientation (where, importantly, the corresponding function for the subsequent vocal responses was virtually flat). Moreover, the slopes of the reaction time functions in Experiment 2 were not significantly different from the post-stimulus rotation slopes obtained in Experiment 1 for the same four stimuli ( 2.71 and $2.21 \mathrm{~ms} / \mathrm{deg}$, respectively) indicating that the physical 
presence of the stimulus being mentally rotated (as in Experiment 1) did not seem to lead to much of an enhancement of the rate of rotation. Interestingly, Cooper also showed that this linear function could extend past 180 degrees by giving both clockwise and counterclockwise rotation instructions along with the advance orientation information. As discussed by Cooper, such a finding does not support the notion that preparation involves orientation specific priming of feature detectors, which should probably depend on the extent of angular departure but not on its direction, but seemingly indicates that the preparatory mental rotation process can pass through a trajectory in a specific requested clockwise and counterclockwise direction.

Effects of Stimulus Complexity and Practice

Further delineating the effect of stimulus complexity on mental rotation rate was the underlying motivation for a study by Bethell-Fox and Shepard (1988). They hypothesized that for novel stimuli, the rate of mental rotation does depend on stimulus complexity but that this dependence will disappear as the stimuli become more familiar. In their first experiment, Bethell-Fox and Shepard simply examined whether the complexity of unfamiliar stimuli can indeed effect the rate of mental rotation. Eight participants participated for two hours each and 18 block patterns differing in complexity were used. The patterns were $3 \times 3$ matrices, with between two and four blocks filled in for each stimulus. Complexity was assessed by the number of distinct pieces within the matrix (between one and three) as well as by number of shaded blocks (between two and four). At the start of each trial, participants were first presented with one of the matrix patterns for inspection. When the participants felt that they had remembered the pattern they pressed a ready button and the pattern was replaced by one of four rotational arrows 
indicating rotations of either 90 or 180 degrees in either a clockwise or counterclockwise direction. When the participants had finished mentally rotating the pattern, they again pressed a ready button, and a test stimulus appeared to which they provided a "same" or "different" manual response, where test patterns could differ either by being in the wrong orientation or by having a different pattern.

In this initial experiment, mental inspection, rotation, and comparison times all increased as stimulus complexity increased. Bethell-Fox and Shepard also found that increasing the angular departure from 90 to 180 degrees led to higher reaction times and that the reaction time differences between these two rotations also increased with increasing stimulus complexity. It was also observed that some of the participants were both more accurate and faster than others with reaction times that depended less on stimulus complexity. This finding led Bethell-Fox and Shepard to speculate that these participants were likely more adept at encoding and subsequently rotating the patterns as integrated wholes as opposed to rotating them piece by piece.

In their second experiment, Bethell-Fox and Shepard (1988) examined the effect of extensive practice on performance in this same task. Eight high school students were chosen that had previously done a battery of psychometric testing three years prior. These participants comprised two groups of four who differed dramatically in scores on three measures of "fluid-visualization" ability (although this group manipulation did not actually turn out to have any significant effects on the reaction time measures). These participants were given 8 to 10 blocks (36 trials each) of initial practice with just the highest and lowest complexity matrix patterns from Experiment 1 . This practice was 
followed by two test blocks that included both the two familiar patterns along with six other new (i.e., unfamiliar) matrix patterns from Experiment 1.

Results indicated that after extensive practice, all reaction times were now quite comparable for the highest and lowest complexity matrix patterns, but significantly slower for the set of six novel matrices. In addition, rotation rates for the highest and lowest complexity matrix patterns were much more comparable $(9.2 \mathrm{vs} 4.8 \mathrm{~ms} / \mathrm{deg}$, respectively) in the test phase of Experiment 2 than they were in Experiment 1 (49.0 vs $1.2, \mathrm{~ms} / \mathrm{deg}$, respectively). Moreover, after omitting two "deviant" participants from the analysis who claimed to be using verbal strategies throughout to encode the locations of the shaded portions of the matrix stimuli, the times taken to rotate the two familiar patterns were essentially identical by the eighth block of practice trials. On the basis of these results, Bethell-Fox and Shepard concluded that if given enough time to practice encoding and mentally rotating patterns of differing complexity, (most) participants were able to develop well-integrated internal representations for them which allow them be rotated in a holistic fashion at similar rates.

A subsequent study that has further served to delineate the effect of practice on the rate of mental rotation was performed by Cohen and Kubovy (1993). In the third experiment of their study, 100 participants performed 48 practice and 720 experimental trials in a single 1-hour session. The task involved the presentation of standard stimulus until the participant indicated that they were ready to proceed, followed by the presentation of a rotated test stimulus to which a "same" or "different" response was required. Of key relevance was the fact that only one 12-sided irregular polygon was always used as the standard stimulus, which was presented in the same fixed orientation 
throughout, and also that the orientations of the test stimuli covered all of the possible orientations from 0 to 359 degrees in 1-degree steps. In addition, half of the participants performed this task under speed pressure.

The results indicated that the mean slope of the reaction time functions for "same" responses (where analogous, but not reported, results were obtained for "different" responses) was $0.94 \mathrm{~ms} / \mathrm{deg}$ under no speed pressure and only $0.55 \mathrm{~ms} / \mathrm{deg}$ under speed pressure. As noted by Cohen and Kubovy, these slope values were either equal to or well below $1 \mathrm{~ms} /$ degree (corresponding to a rotation rate of $1000 \mathrm{deg} / \mathrm{s}$ ), a value which they had previously argued represented the shallowest allowable slope which could reasonably be considered to be the result of a mental rotation process. These researchers concluded that under conditions involving a very small set of standard stimuli, participants were able to form an orientation-free representation of the standard stimulus, that nonetheless still contains the handedness information that allows them to accurately perform the same-different task without using mental rotation. Importantly, the fact that all possible 360 degree test rotations were used allowed Cohen and Kubovy to rule out the possibility that participants might have been able circumvent the mental rotation process by memorizing the shape of the standard within all possible test orientations. Moreover, an examination of the actual reaction time versus angular distance plots themselves did not reveal the presence of any cyclical patterns that would have suggested the participants had memorized a smaller set of prototype orientations (c.f., Tarr \& Pinker, 1989). Effects of Stimulus Dimensionality and Type of Task

Previous research had shown that mental rotation rates are typically much higher when comparing the shapes of two three-dimensional (Shepard \& Metzler, 1971) cubical 
block figures presented simultaneously than when comparing the shape of a single twodimensional alphanumeric or random polygon test figure to a learned internal standard. Hence, the extent to which these differences were due to the effect of either stimulus dimensionality or type of task was examined by Shepard and Metzler (1988). With respect to stimulus dimensionality, Shepard and Metzler proposed that it might be more difficult to construct an internal representation for a three-dimensional object than for a two-dimensional one. If so, more time would be required to initially encode threedimensional objects and, additionally, more processing might also be required when mentally rotating them. With respect to the type of task, Shepard and Metzler proposed that mental rotation might occur more slowly when two stimuli are simultaneously presented for comparison (typically, in different, unpredictable overall orientations across trials) than when only one test stimulus is presented and compared to a standard learned in one particular orientation because, in the former case, participants might have trouble maintaining representations for both of the stimuli while performing the mental rotation and, hence, tend to look back and forth between them.

In their study, 26 participants performed two 45-min experimental sessions within which two- and three-dimensional stimuli were compared, respectively, with 13 participants performing each type of task. For both types of tasks, specific instructions to mentally rotate were given. Each session consisted of 80 (i.e., 40 same and 40 different under reflection) test trials involving orientation differences of 0 to 180 degrees in 45 degree increments in either direction. The two-dimensional stimuli were based on a single random 24-point polygon and the three-dimensional ones on a single figure made 
up of seven blocks with two right angles (which was a somewhat simplified figure than those used in previous studies).

Because Shepard and Metzler argued that reaction times for "different" responses were difficult to interpret given that negative responses can sometimes be based on shortcut non-rotational strategies, they reported results for correct "same" responses only. These results indicated that although the intercepts of the reaction time versus angular disparity functions (and, hence, the overall reaction times themselves) were higher for the three-dimensional objects than for the two-dimensional ones, the slopes of these functions within each task type were nearly the same. From these findings, Shepard and Metzler concluded that although more time is required to initially encode threedimensional objects into memory (and, perhaps, also to search for relevant segments of the object when determining its starting orientation and to confirm the "same-different" response once rotated), dimensionality does not then effect the rate of mental rotation.

Type of task, though, was found to significantly effect the rate of mental rotation in that slopes of 6.4 and $7.7 \mathrm{~ms} / \mathrm{deg}$, respectively, were obtained for the two- and threedimensional objects when two simultaneously presented stimuli had been compared, and slopes of 2.1 and $2.9 \mathrm{~ms} / \mathrm{deg}$, respectively, were obtained for comparisons involving a single test stimulus (i.e., a threefold difference in rotation rate). Shepard and Metzler attributed these slope differences either to the fact that multiple repeated back-and-forth comparisons are occurring during the rotation or to the fact that some piece-by-piece rotation is taking place when comparing two simultaneously presented stimuli.

Subsequently though, Bauer and Jolicoeur (1996) proposed that Shepard and Metzler's (1988) null results with respect to dimensionality differences in rotation rate 
were due to the fact that their two- and three-dimensional stimuli were biased towards being relatively difficult and relatively simplified, respectively. Hence, in Bauer and Jolicoeur's study two- and three-dimensional cubical 10-block stimuli with a varying number of right-angle bends (i.e., two, three, or four) were generated by taking each of six such two-dimensional stimuli and turning one of their arms out of the twodimensional plane (thus rendering the stimuli directly comparable across dimensionalities). In Experiment 1, each of these 12 stimuli was simultaneously presented for comparison along with either itself or its mirror-image reflection (paired horizontally). In Experiment 2, only stimuli with three right-angle bends were used. Angular offsets ranged from 0 to 180 degrees in 30 degree increments in the depth plane for Experiment 1 and in both the depth and picture planes for Experiment 2.

Twelve participants performed 840 trials with both types of stimuli in one $90-$ min session for Experiment 1. The key finding in this experiment was the presence of an Orientation $\times$ Dimensionality interaction in the correct reaction times for "same" responses. Namely, they found that the rotation rates were $82 \mathrm{deg} / \mathrm{s}$ (i.e., $12.2 \mathrm{~ms} / \mathrm{deg}$ ) for the two-dimensional objects but only $55 \mathrm{deg} / \mathrm{s}$ (i.e., $18.2 \mathrm{~ms} / \mathrm{deg}$ ) for the threedimensional objects. To ensure that these results were reliable, 32 more participants performed 630 trials in each of two 2-hour experimental sessions in Experiment 2. Half of them performed depth rotations and half performed picture-plane rotations. Again, the key finding was the presence of an Orientation $\times$ Dimensionality interaction in the correct reaction times for "same" responses such that the rotation rates were $86 \mathrm{deg} / \mathrm{s}$ (i.e., 11.6 $\mathrm{ms} / \mathrm{deg}$ ) for the two-dimensional objects but only $76 \mathrm{deg} / \mathrm{s}$ (i.e., $13.2 \mathrm{~ms} / \mathrm{deg}$ ) for the three-dimensional objects. Bauer and Jolicoeur concluded that such findings were 
consistent with the notion that objects represented in three dimensions have a higher informational load than those represented in only two dimensions, making any mental transformations performed on them more difficult.

Reaction Time Distributions

Much previous research supports the notion that people can mentally rotate visual images. The presence of a linear relation between reaction time and angle of rotation in mental rotation comparison tasks is a finding that has now been replicated quite extensively. However, the issue of whether this linear relationship exists for all of the individual reaction times or simply just for the mean reaction times is one that has not yet been determined. Hence, in this study the relation between the shapes of the resulting reaction time distributions as a function of the angle of rotation within this paradigm will be examined.

Previously, Hockley (1984) used a similar approach in his work involving visual and memory search processes. In Hockley's Experiment 1, there were two tasks. The first was a visual search task that required participants to search for a target letter from a list of between 3-6 items presented visually in a vertical list. The second was a memory search task that required participants to recall from memory whether or not a target letter appeared on a previously seen list of between 3-6 items. For both types of search tasks, mean reaction times increased linearly with item set size. Importantly, Hockley then went on to fit the ex-Gaussian distributional model to the reaction time distributions at each set size. This model provides three parameters that index the location of the leading edge or mode (mu), the amount of spread at the front end of the distribution (sigma), and the size of the tail (tau). This analysis showed that for the visual search task, the increases in 
mean reaction time with set size were largely reflected by an increases in the ex-Gaussian parameter mu (although this was demonstrated by Hockley only for negative trials and positive trials involving the last item on the list). This result was then interpreted as indicating that visual search proceeds in a serial fashion through the list whereby increasing the set size increases the number of additional items that need to be searched on each trial (i.e., which should then shift the leading edge of the reaction time distributions). In the memory search task, Hockley found that both the mu and tau exGaussian parameters increased as a function of the set size of remembered items (for all positive and negative trials). However, it was tau that increased much more so than mu.

Hence, a clear dissociation between the results obtained in each task with respect to the two ex-Gaussian parameters mu and tau was obtained by Hockley. For the visual search task, it was the leading edge of the distribution (i.e., mu) that increased with set size, whereas for the memory search task, the distribution tended to become more positively skewed with increases in set size (i.e., the size of the tail end of the distribution, as indexed by the value of tau, increased). As argued by Hockley (1984) such a result was clearly not consistent with a serial search of the memory set items. Ex-Gaussian

When a distribution is symmetrical, its central tendency can be measured accurately with standard methods of analysis (mean, median and mode). However, when a distribution is skewed (as reaction distributions typically are), these standard methods do not compliment one another and another form of analysis is needed rather then simply trimming or transforming the data. Using a distributional analysis like the ex-Gaussian 
preserves all of the available data and provides a clearer description of the reaction time phenomena of interest.

The motivation for using ex-Gaussian analysis also stems from the notion that the three parameters (mu, sigma and tau) can help to identify the different cognitive processes that are invoked when performing any particular cognitive task. For example, as just mentioned, Hockley (1984) found that the use of the ex-Gaussian analyses provided more explicit results and aided conclusions regarding whether or not a serial search process could be inferred.

The present paper will involve a similar analysis, namely it will examine whether the linear increase in mean reaction times in the mental rotation paradigm is associated with increases in the ex-Gaussian mu or tau parameters. If the previous conclusions about mental rotation are correct, increases in the mu parameter should be found as the angle of orientation increases, analgous to Hockley's (1984) finding that mu increases with set size in the visual search task. In other words, if the linear increases in mean reaction times do reflect actual analog properties of image rotation (i.e., greater rotation distances), then such increases should be reflected in all reaction times, thereby shifting the reaction time distributions further along the reaction time scale as a whole (where differences in reaction time distribution locations are typically indexed by differences in $\mathrm{mu})$. As a concrete example, consider physically rotating an object either 60 or 160 degrees. Although some of the 160 degree rotations could be done as fast as some of the 60 degree rotations, it is not logically possible for the fastest 160 degree rotation time to be as fast as the fastest 60 degree rotation time.

Method 


\section{Participants}

Twenty-two Carleton University students participated in this experiment. All participants were drawn from the Carleton University participant pool and were run individually in $1 \frac{1}{2}$-hour sessions. Participants received partial hours credit towards a first-year psychology course.

\section{Stimuli and Apparatus}

One of three capital letters (F, L, and P) in either their standard or reflected, (i.e., mirror-image) form were presented on each trial. The stimuli appeared in either their upright position or in varying 30 degree increments from 0-330 degrees (i.e., 0,30 , $60 \ldots)$. The letters were $3 \mathrm{~cm}$ tall on the computer screen and were presented in black against a white background. The experiment was programmed and run on a Pentium IV computer with Superlab 2.0. Responses were given via a Microsoft serial mouse with the roller ball disabled.

Procedure

Participants sat approximately two feet away from the computer screen. They were told to complete each trial as quickly as possible while still trying to keep errors to a minimum. Before the onset of a letter stimulus, a plus sign appeared as a fixation point at the center of the screen for $1000 \mathrm{~ms}$ followed by a blank screen for $500 \mathrm{~ms}$. The presentation of the letter stimulus followed until a response was given. Between each trial, a $1000 \mathrm{~ms}$ blank screen was presented. Participants were instructed to respond "Yes" if they believed that the letter had been presented in its standard form or "No" if they believed that it had been presented in its reflected (or mirror-image) form. "Yes" and "No" responses were made via the left and right buttons on the serial mouse, respectively 
(which was always held in the right hand positioned on the table). Participants performed 12 blocks of 84 randomized trials with the first 2 blocks regarded as practice trials (resulting the collection of 840 non-practice trials per participant). Within each block, each letter and its mirror image were shown once at each rotation except for 0 and 180 degrees for which they were shown twice per block.

Results

\section{Mean Reaction Time and Accuracy}

The dependent measures to be analyzed in these initial analyses were the mean correct reaction times and the arcsine transformed mean proportion correct for each cell in the following design. Before performing the analyses, 2 of the 22 participants were dropped, one of whom made 229 errors during the last 10 blocks of test trials (where the next 5 most error-prone participants made 94, 91, 65, 43, and 39 errors, respectively) and the other of whom had a very slow mean correct reaction time of $2142 \mathrm{~ms}$ for those same blocks (where the next 5 slowest participants had mean correct reaction times of 1631 , $1388,1262,1188$, and $1112 \mathrm{~ms}$, respectively). In addition, all of the data from the first 2 blocks of practice trials were discarded and any reaction times that were either above 3 $S D$ s of a participant's mean correct reaction time (223 in total or $1.3 \%$ ) or below $150 \mathrm{~ms}$ ( 3 in total) were also removed from the data.

The five within-participant (or repeated measures) independent variables specified in these analyses were stimulus type ( 2 levels: standard and reflected), blocks ( 2 levels: first five and last five), letters (3 levels: F, L, and P), direction (2 levels: clockwise and counter-clockwise), and orientation (7 levels: $0,30,60,90,120,150$, and 180 degrees). For the mean reaction time analysis, a 2-level between-participant independent variable 
was also specified which simply involved a median split classification of the 10 fastest and 10 slowest participants. Although this latter factor was not included in the accuracy analyses, note that overall accuracy was somewhat lower for the 10 fastest participants (i.e., $96.06 \%$ ) than for the 10 slowest participants (97.42\%). All of the statistical significance results reported here were based on the more conservative, GreenhouseGeisser epsilon adjusted degrees of freedom (although the reported degrees of freedom themselves are the unadjusted ones). For the reader's convenience, a summary of all of the significant effects found in the ANOVA analysis of the mean correct reaction time is given in Table 1.

Mean Reaction Time. The main effect of stimulus type was significant, $F(1,18)=$ 45.042, $p<.001, M S E=227004$. Mean reaction times were faster for letter stimuli presented in their standard form than in their reflected form (842 vs. $952 \mathrm{~ms}$, respectively, for correct "Yes" and "No" responses). The main effect of blocks was also significant, $F(1,18)=16.958, p<.005, M S E=423747$. Mean reaction times were slower in the first five blocks of testing than in the second five ( $943 \mathrm{vs.} 850 \mathrm{~ms}$, respectively). The main effect of letters was also significant, $F(2,36)=3.870, p<.05$, $M S E=129114$. Marginal mean reaction times for the letters $\mathrm{F}, \mathrm{L}$, and $\mathrm{P}$ were 880,920 , and $890 \mathrm{~ms}$, respectively. The main effect of direction was also significant, $F(1,18)=$ $8.786, p<.01, M S E=73009$. Marginal mean reaction times for clockwise and counterclockwise rotations were 910 and $883 \mathrm{~ms}$, respectively (note that in the experimental code itself, one of each of the two replicated responses per letter and stimulus type for each of the 0 and 180 degree rotations was arbitrarily assigned to each direction within each block). Finally, and most importantly, the main effect of orientation was also 
significant, $F(6,108)=62.492, p<.001, M S E=185001$. Polynomial contrast analyses indicated that both the linear and the quadratic trends of the orientation effect were significant $(F[1,18]=81.994, p<.001, M S E=824448$, and $F[1,18]=11.434, p<.005$, $M S E=149768$, respectively). Marginal mean reaction times for each of the $0,30,60,90$, 120,150 , and 180 degree angles of orientation are reported in Table 2.

Stimulus type interacted significantly with letters $(F[2,36]=9.867, p<.005$. $M S E=40728$; i.e., mean reaction times for the letters $\mathrm{F}, \mathrm{L}$, and $\mathrm{P}$ were 817,887 , and 821 ms, respectively, for standard stimuli but 943,954 , and $959 \mathrm{~ms}$, respectively, for reflected stimuli), direction $(F[1,18]=9.747, p<.01 . M S E=19248$; i.e., the mean reaction times for the clockwise and counter-clockwise rotations were 863 and $820 \mathrm{~ms}$, respectively, for standard stimuli but 958 and $945 \mathrm{~ms}$, respectively, for reflected stimuli), and orientation $(F[6,108]=4.659, p<.01, M S E=51943$, with $F[1,18]=14.166, p<.005, M S E=$ 62763 , and $F[1,18]=4.624, p<.05, M S E=117088$, for the linear and quadratic trends of this interaction, respectively; mean reaction times for each stimulus type at each angle of orientation are also reported in Table 2 where essentially the slope of the reaction time vs. orientation function is steeper for correct "Yes" responses to standard stimuli and slightly more curved). There were also significant three-way interactions involving both stimulus type, letters, and direction, $F(2,36)=4.997, p<.05, M S E=71150$, and stimulus type, letters, and orientation, $F(12,216)=2.559, p<.05, M S E=26281$.

Blocks interacted significantly with both direction $(F[1,18]=4.749, p<.05$. $M S E=11838$; i.e., the mean reaction times for the clockwise and counter-clockwise rotations were 961 and $925 \mathrm{~ms}$, respectively, for the first five testing blocks but 860 and $841 \mathrm{~ms}$, respectively, for the second five testing blocks $)$ and orientation $(F[6,108]=$ 
$3.242, p<.05, M S E=25629$, with $F[1,18]=8.504, p<.01, M S E=38865$, for the linear trend of this interaction; mean reaction times for each half of the testing blocks at each angle of orientation are also reported in Table 2 where essentially the slope of the reaction time vs. orientation function is steeper for the first five than for the last five blocks of trials). There was also a significant three-way interaction involving blocks, stimulus type, and direction, $F(1,18)=5.344, p<.05, M S E=19462$. Perhaps somewhat interestingly, however, neither the two-way Blocks $\times$ Stimulus Type interaction, $F(1,18)$ $=0.011, p>.90, M S E=18556$, nor the three-way Blocks $\times$ Stimulus Type $\times$ Orientation interaction, $F(6,108)=0.475, p>.75, M S E=32171$, were significant indicating that neither the overall differences nor the reaction time versus angle of orientation slope differences between correct "Yes" and "No" responses changed from the first half to the last half of the experimental session.

Not surprisingly, the between-participant factor that contrasted the 10 fastest and 10 slowest participants was significant, $F(1,18)=28.702, p<.001, M S E=41083461$, where the mean correct reaction times for these two groups were 709 and $1084 \mathrm{~ms}$, respectively. This factor also entered into significant two-way interactions with stimulus type $(F[1,18]=12.001, p<.005, M S E=227004$; i.e., the mean reaction times for the standard and reflected stimuli were 683 and $736 \mathrm{~ms}$, respectively, for the 10 fastest participants but 1000 and $1168 \mathrm{~ms}$, respectively, for the 10 slowest participants), blocks $(F[1,18]=4.624, p<.05, M S E=423747$; i.e., the mean reaction times for the first and last five blocks were 731 and $687 \mathrm{~ms}$, respectively, for the 10 fastest participants but 1154 and $1014 \mathrm{~ms}$, respectively, for the 10 slowest participants), and orientation $(F[6$, $108]=8.026, p<.005, M S E=185001$, with $F[1,18]=10.715, p<.005, M S E=824448$, 
for the linear trend of this interaction; mean reaction times for each group of participants at each angle of orientation are also reported in Table 2 where essentially the slope of the reaction time vs. orientation function is steeper for the slowest group than for the fastest group). No higher order interactions involving this factor were significant.

Accuracy. The main effect of stimulus type was significant, $F(1,19)=21.491, p$ $<.001, M S E=0.185$. Accuracy was lower for letter stimuli presented in their standard form than in their mirror-image form ( .952 vs. .983 , respectively). The main effect of blocks was also significant, $F(1,19)=5.437, p<.05, M S E=0.072$. Accuracy was higher in the first five blocks of testing than in the second five (.971 vs. .963 , respectively). The main effect of letters was also significant, $F(2,38)=7.741, p<.005$, $M S E=0.32$. The proportion correct for the letters $\mathrm{F}, \mathrm{L}$, and $\mathrm{P}$ were $.967, .961$, and .974 , respectively. Finally, the main effect of orientation was also significant, $F(6,114)=$ $19.202, p<.001, M S E=0.152$. Proportion correct for each of the $0,30,60,90,120,150$ and 180 degree angles of orientation are reported in Table 3.

Stimulus type interacted significantly with letters $(F[2,38]=5.225, p<.05 . M S E$ $=0.067$; i.e., proportion correct for the letters $\mathrm{F}, \mathrm{L}$, and $\mathrm{P}$ were $.951, .938$, and .966 , respectively, for standard stimuli but $.983, .983$, and .982 , respectively, for reflected stimuli) and orientation $(F[6,114]=14.066, p<.001, M S E=0.127$; proportion correct for each stimulus type at each angle of orientation are also reported in Table 3). Note that the fact that accuracy was actually much higher for the reflected than for the standard stimuli at 180 degree rotations indicates that the fact that the mean correct reaction times for the reflected and standard stimuli are almost equal for that orientation cannot possibly be due to a speed-accuracy tradeoff in the responding to the 180-degree reflected stimuli. 
Letters also interacted significantly with orientation $(F[12,228]=3.366, p<.005, M S E=$ 0.050; proportion correct for each letter at each angle of orientation are also reported in Table 3).

There were also significant three-way interactions involving stimulus type, letters, and direction $(F[2,38]=8.012, p<.005, M S E=0.024)$, stimulus type, letters, and orientation $(F[12,228]=6.240, p<.001, M S E=0.041)$, and stimulus type, direction, and orientation $(F[6,114]=2.566, p<.05, M S E=0.037)$. Blocks only entered into two fourway interactions involving blocks, stimulus type, letters, and direction $(F[2,38]=3.501$, $p<.05, M S E=0.036)$ and blocks, letters, direction, and orientation $(F[12,228]=2.842$, $p<.05, M S E=0.028)$.

\section{Ex-Gaussian Parameters}

The dependent measures that were analyzed in these subsequent analyses were each of the three ex-Gaussian parameters mu, sigma, and tau. The three withinparticipant independent variables used for these analyses were stimulus type (2 levels: standard and mirror image), blocks ( 2 levels: first five and last five), and orientation (7 levels: $0,30,60,90,120,150$, and 180 degrees). Values for each of the three distributional parameters were obtained by fitting the ex-Gaussian distribution to the set of correct reaction time data collected for each individual participant within each cell of this design (i.e., $20 \times 28=540$ fits in total).

Note that in order to fit the ex-Gaussian distribution, a decent amount of observations in each cell needed to be available for each participant. Hence, it was necessary to aggregate together the correct reaction times for each of the letters (i.e., F, L, and $\mathbf{P}$ ) and each direction of rotation (i.e., clockwise and counter-clockwise) within each 
of those aforementioned cells. This move then provided 30 observations per fit (i.e., 6 "Yes" and 6 "No" responses per block for each of $0,30,60,90,120,150$, and 180 degrees of rotation) minus any error responses. Although the main effects of each of these two latter factors had been significant in the previous analysis of mean reaction times, the actual mean differences were deemed small enough so as not to make this aggregation too worrisome. In addition, none of the interactions involving either of these two factors and orientation were significant in that previous analysis (i.e., Letters $\times$ Orientation, $F[2,216]=1.704, p>.15, M S E=30665$; Direction $\times$ Orientation, $F[6,108]$ $=0.648, p>.50, M S E=35612$; and Letters $\times$ Direction $\times$ Orientation, $F[2,216]=1.491$, $p>.20, M S E=24549$; and note also that the Letters $\times$ Direction interaction, $F[2,36]=$ $0.041, p>.90, M S E=39230$, itself was not significant).

The software used to perform the ex-Gaussian fits made use of a quantile maximum likelihood estimation (QMLE; Brown \& Heathcote, 2003; Heathcote, Brown, \& Cousineau, 2004) fitting procedure. A descriptive example of what quantiles are and how this procedure uses them to fit the ex-Gaussian is provided in the Appendix. This program provides output exit codes that indicate whenever there was a problem with one of the ex-Gaussian fits (Brown, Cousineau, \& Heathcote, 2004). With respect to these codes, the developers explicitly state that "In our experience, any exit code smaller than 32 means that both the parameter estimates and their standard errors and correlations are trustworthy" (p. 4), with mainly the latter two measures becoming unstable with higher exit codes. For this present set of data, good fits (i.e., with very small exit codes of either 1 or 3) were obtained for all of the 540 distributions except 7 (for each of which sigma was estimated to be 0 or very near 0 ). These fits were obtained by first specifying that 19 
quantiles were to be estimated and fit for each of the 540 distributions, and then by adjusting the number of quantiles used in the fitting procedure for each of the 89 distributions that didn't converge to an ex-Gaussian fit using 19 quantiles (which included 5 distributions for which less than 20 reaction times were actually available to be fit).

In the ANOVA analyses of the values for each of the three parameters that were obtained for each distribution, as in the previous mean reaction time analysis, the 2-level between-participant independent variable contrasting the 10 fastest and 10 slowest participants was also included (along with the three within-participant stimulus type, blocks, and orientation factors). All of the statistical significance results reported here were based on the more conservative, Greenhouse-Geisser epsilon adjusted degrees of freedom (although the reported degrees of freedom themselves are the unadjusted ones). For the reader's convenience, a summary of all of the significant effects found in these three analyses is given in Table 4.

All of the cell means for this design are shown in Figures $1 \mathrm{~A}$ and 1B along with the corresponding mean correct reaction times. As well, Figures 2A and 2B show representative group reaction time distributions for the responses of the 10 fastest and the 10 slowest participants, respectively, obtained in the last five blocks to stimuli presented in their standard form. In this figure, these distributions are plotted as fixed-area, quantile-based, histogram bins along with the corresponding best-fitting, theoretical exGaussian curves. The group quantile values were obtained by averaging the $10^{\text {th }}, 20^{\text {th }}$, $30^{\text {th }}$, etc. quantile values (calculated from the raw RT data by QMLE) across participants 
at each orientation (but note that the $0^{\text {th }}$ and $100^{\text {th }}$ quantiles for each orientation were estimated by the average minimum and maximum values, respectively).

$M u$. The main effect of stimulus type was significant, $F(1,18)=73.541, p<.001$, $M S E=9384 . \mathrm{Mu}$ was lower for letter stimuli presented in their standard form than in their reflected form (592 vs. $662 \mathrm{~ms}$, respectively, for correct "Yes" and "No" responses). The main effect of blocks was also significant, $F(1,18)=27.905, p<.001, M S E=$ 12432. Mu was higher in the first five blocks of testing than in the second five (652 vs. $602 \mathrm{~ms}$, respectively). The main effect of orientation was also significant, $F(6,108)=$ $53.185, p<.001, M S E=18054$. Polynomial contrast analyses indicated that the linear, quadratic, and cubic trends of the orientation effect were significant $(F[1,18]=75.024, p$ $<.001, M S E=69093, F[1,18]=28.668, p<.001, M S E=17392$, and $F[1,18]=7.318, p$ $<.014, M S E=7893$, respectively). Marginal mean values of mu for each of the $0,30,60$, $90,120,150$, and 180 degree angles of orientation are reported in Table 5 (and see also Figures 1A and 1B).

Blocks interacted significantly with orientation $(F[6,108]=4.614, p<.01, M S E$ $=3268$, with $F[1,18]=8.591, p<.01, M S E=3533$, and $F[1,18]=13.231, p<.005$, $M S E=2287$, for the linear and quadratic trends of this interaction; mean values of mu for each half of the testing blocks at each angle of orientation are also reported in Table 5 where essentially both the slope and the curvature of the mu vs. orientation function are smaller for the last five blocks of trials). Again not surprisingly, the between-participant factor that contrasted the 10 fastest and 10 slowest participants was significant, $F(1,18)=$ $12.325, p<.01, M S E=247763$, where the mean values of mu for these two groups were 553 and $701 \mathrm{~ms}$, respectively. This factor also entered into significant two-way 
interactions with both stimulus type $(F[1,18]=21.109, p<.001, M S E=9283$; i.e., mean values of mu for the standard and reflected stimuli were 537 and $570 \mathrm{~ms}$, respectively, for the 10 fastest participants but 647 and $755 \mathrm{~ms}$, respectively, for the 10 slowest participants $)$ and orientation $(F[6,108]=6.708, p<.001, M S E=18054$, with $F[1,18]=$ $8.030, p<.05, M S E=69093$, and $F[1,18]=6.047, p<.05, M S E=17392$, for the linear and quadratic trends of this interaction, respectively; mean values of mu for each group of participants at each angle of orientation are also reported in Table 5 where essentially both the slope and the curvature of the mu vs. orientation function are smaller for the fastest group of participants).

Sigma. The main effect of stimulus type was significant, $F(1,18)=14.316, p<$ $.005, M S E=3206$. Sigma was lower for letter stimuli presented in their standard form than in their reflected form (73 vs. $91 \mathrm{~ms}$, respectively, for correct "Yes" and "No" responses). The main effect of orientation was also significant, $F(6,108)=41.084, p<$ $.001, M S E=2654$. Polynomial contrast analyses indicated that the linear and quadratic trends of the orientation effect were significant $(F[1,18]=90.886, p<.001, M S E=$ 5871 , and $F[1,18]=27.942, p<.001, M S E=3392$, respectively). Marginal mean values of sigma for each of the $0,30,60,90,120,150$, and 180 degree angles of orientation are reported in Table 6 (and see also Figures 1A and 1B).

The between-participant factor that contrasted the 10 fastest and 10 slowest participants was significant, $F(1,18)=15.977, p<.005, M S E=13586$, where the mean values of sigma for these two groups were 62 and $107 \mathrm{~ms}$, respectively. This factor also entered into a significant two-way interaction with orientation $(F[6,108]=7.716, p<$ $.001, M S E=2654$, with $F[1,18]=14.271, p<.005, M S E=5871$, and $F[1,18]=5.095, p$ 
$<.05, M S E=3392$, for the linear and quadratic trends of this interaction, respectively; mean values of sigma for each group of participants at each angle of orientation are also reported in Table 6 where essentially both the slope and the curvature of the sigma vs. orientation function are smaller for the fastest group of participants).

Tau. The main effect of stimulus type was significant, $F(1,18)=5.838, p<.05$, $M S E=41768$. Tau was lower for letter stimuli presented in their standard form than in their reflected form (252 vs. $293 \mathrm{ms,}$, respectively, for correct "Yes" and "No" responses). The main effect of blocks was also significant, $F(1,18)=5.932, p<.05, M S E=44648$. Tau was higher in the first five blocks of testing than in the second five (294 vs. $251 \mathrm{ms,}$ respectively). The main effect of orientation was also significant, $F(6,108)=11.628, p<$ $.001, M S E=18745$. Polynomial contrast analyses indicated that only the linear trend of the orientation effect was significant $(F[1,18]=28.927, p<.001, M S E=41800)$. Marginal mean values of tau for each of the $0,30,60,90,120,150$, and 180 degree angles of orientation are also reported in Table 6 (and see also Figures 1A and 1B).

The between-participant factor that contrasted the 10 fastest and 10 slowest participants was significant, $F(1,18)=23.756, p<.001, M S E=315071$, where the mean values of tau for these two groups were 157 and $388 \mathrm{~ms}$, respectively. This factor also entered into a significant two-way interaction with orientation $(F[6,108]=4.440, p<$ $.005, M S E=18745$, with $F[1,18]=5.719, p<.05, M S E=41800$, and $F[1,18]=7.393, p$ $<.05, M S E=26093$, for the linear and quadratic trends of this interaction, respectively; mean values of tau for each group of participants at each angle of orientation are also reported in Table 6 where essentially both the slope and the curvature of the tau vs. orientation function are smaller for the fastest group of participants - note that this 
relation is essentially flat from $0-150$ degrees for the fastest group). Apparently, this between-participant factor did not significantly interact with stimulus type, $F[1,18]=$ $1.230, p>.25, M S E=41768$, even though the differences between the measures of tau for standard and reflected stimuli seem to be less for the fastest than for the slowest group of participants in Figures 1A and 1B.

\section{Discussion}

The results obtained here indeed indicate that the mean correct reaction time to make a standard or reflected comparison response to a rotated letter increases with its angle of rotation. As is evident in Figures $1 \mathrm{~A}$ and $1 \mathrm{~B}$, the nature of this relation is clearly curvilinear for both standard and reflected comparison responses (i.e., "Yes" and "No" responses, respectively), although it is somewhat less steep and less curved for the responses to the reflected stimuli. Moreover, it is also evident that this curvilinear relation held for both the 10 slowest and the 10 fastest groups of participants, although it is somewhat less steep for the fastest group.

Of key interest in this present work is the extent to which these relations are present in the three ex-Gaussian distributional parameters for the "Yes" responses to the stimuli presented in their standard form. To help address this question, the slopes of the mental rotation functions for each reaction time measure are given in Table 7 for each of the first and last five blocks and for each of the 10 slowest and 10 fastest groups of participants. Given the inherent nonlinearity that is present in these functions, slope values are reported separately for both the $0-90$ degree and the $90-180$ degree sections. For the slowest group of participants, it is clear that the linear increases in mean correct reaction times between 0 and 90 degrees for both the first five and last five blocks 
are accompanied by linear increases in both mu and tau (but more so for tau) and no increase in sigma. For this group, the linear increases in mean correct reaction times between 90 and 180 degrees are accompanied by linear increases in both mu and tau (but now more so for mu) and now also in sigma. On the other hand, for the fastest group of participants, it is clear that the linear increases in mean correct reaction times between 0 and 90 degrees for both the first five and last five blocks are accompanied by linear increases in mu only. For this group, the linear increases in mean correct reaction times between 90 and 180 degrees are accompanied by linear increases in both mu and tau (but much more so for mu) and now also in sigma. In any case, it seems to be clear from these slope results, that something different seems to be going on with respect to the manner in which responses are being generated to standard stimuli at orientations below 90 degrees and above 90 degrees.

With respect to the issue of whether these distributional analyses indicate whether or not mental rotation is occurring, it is informative to look what is happening to the shapes of the reaction time distributions where the mental rotation function is the flattest, namely, the $0-90$ degree section in the last five blocks for the fastest group of participants. Here, it is clear that the reaction time distributions are simply shifting higher but not changing in shape given that mu is increasing but both sigma and tau are not (see also Figure 2A). In some sense, it could be argued that such an almost perfect shift in these distributions is highly indicative that a mental rotation process is indeed taking place, albeit one that is occurring at very fast (i.e., efficient) rate. Nonetheless, some other possibilities can also be considered. 
Nonlinearity. In general, nonlinearity of mental rotation functions is typically found only for highly familiar stimuli such as letters (e.g., Cooper, 1975; Hock \& Tromley, 1978; Koriat \& Norman, 1985). To account for such a finding, Hock and Tromley (1978) hypothesized that mental rotation does not actually have to be invoked for familiar stimuli that are presented in orientations for which they could still be regarded as being perceptually upright. More specifically, if the top and bottom position of a test stimulus is consistent with that encoded in memory, it is deemed upright (Rock \& Heimer, 1957) and a judgment as to whether or not it is reflected can be made without having to rotate it. In their mental rotation study, nine participants performed three onehour sessions. Three sets of two test stimuli each were used, the first of which consisted of circular letters $(e, G)$, the second of elongated letters $(L, J)$, and the third of more standard rectangular shapes $(\mathrm{R}, \mathrm{F})$. The stimuli were rotated in varying degrees by increments of 36 degrees. Their results indicated that the degree of nonlinearity in the mental rotation functions depended on the stimulus set in that it was linear only for the first circular set (for which top and bottom could presumably only be defined at a 0 degree of rotation) and quite nonlinear for the second elongated set (i.e., the function was flat up to a 72 degree rotation). The intermediate degree of curvilinearity in the mental rotation function for their third set of rectangular letters was quite similar to that obtained in the present study and would likely have to be interpreted by Hock and Tromley (1978) as indicating that these types of stimuli are indeed being rotated even at smaller angles of orientation by at least some of the participants. If not, the initial parts of mental rotation function for the reaction times in this study, and likely also for each of the three exGaussian parameters as well, should essentially have been flat. 
The issue of nonlinearity in the mental rotation functions obtained for highly familiar stimuli was also examined by Koriat and Norman (1985) who proposed that extensive practice with a visual stimulus results in a broadly tuned visual memory representation which then allows for automatic recognition of that stimulus over a range of orientation offsets around the upright position. In their Experiment 1, 16 Hebrew students made normal or reflected judgments involving four Hebrew letters that were rotated in varying 60-degree increments. They found that the mental rotation functions were steeper and more nonlinear for the standard than for the reflected stimuli (findings which were essentially replicated in the present study). According to Koriat and Norman (1985), such results make perfect sense because participants would certainly have had much more previous practice perceiving standard rather than reflected versions of letters and, hence, would be more likely to have a broadly tuned visual memory representation for the former than for the latter (although they also went on to show in their next experiment that mental rotation functions can become somewhat nonlinear for reflected stimuli as well as participants gain some experience processing them). Note, that the notion of a broadly tuned memory representation could be regarded as being consistent with the presence of slight increases in the mental rotation function from $0-90$ degrees given that such tuning is likely to become less sensitive as the angle of orientation increases. Nonetheless, it could also be expected that gradual decreases in tuning sensitivity (and, hence, the clarity of the recognition process) be accompanied by increases in both mu and tau whereas, for the fastest group of participants at least, only $\mathrm{mu}$ increased across for $0-90$ rotations. 
Standard and Reflected Stimuli. In general, it is invariably found that reaction times to reflected stimuli are slower than for standard stimuli (Cooper, 1975; Hock \& Tromley, 1978; Koriat \& Norman, 1982), as is indeed the case in this present study. However, it is also the case, as it is here, that mental rotation functions for letter stimuli (unlike for other types of 2-D random polygon or 3-D block stimuli) are shallower for reflected than for standard stimuli (Hock \& Tromley, 1978; Koriat \& Norman, 1982), even to the extent that the functions tend to converge together by the 180-degree orientation. Such a finding is important because it indicates that, at least for these familiar stimulus types, reflected responses aren't simply result of an additional processing step (e.g., mentally flipping a "Yes" response to a "No" response if the rotated stimulus does not match it's standard form) because that should then have resulted in a constant increment to the reaction times at each angle of orientation. Hence, it also seems like there might be something different going on with the processing involved in responding to the standard stimuli and the reflected stimuli.

One interesting aspect of these present data that could shed light on this issue is that, for the fastest group of participants at least, both the mean reaction time results and those for all three ex-Gaussian parameters are very similar for both standard and reflected stimuli for orientations of 120 - 180 degrees (especially for the last five blocks of trials). This finding suggests that the processing that is occurring for these faster participants for these orientations might actually be very similar for both types stimuli, where two possibilities exist with respect to the nature of this processing (both of which might be contributing). First, it is possible that explicit mental rotation is indeed required for orientations beyond 90 degrees for both standard and reflected stimuli (where for 
orientations below that, some direct recognition is possible for both stimuli types whenever enough practice with each of them has occurred). Second, it has been suggested that judgements involving reflected stimuli involve a more extensive checking process (Koriat \& Norman, 1985), hence it is possible that these participants are also invoking such a process for judgments involving standard stimuli that have been rotated more than 90 degrees.

For the slower group of participants, however, the pattern of the mean reaction times to standard and reflected stimuli does indeed look quite additive (although it also looks like the increases in mu at higher orientations are being offset by corresponding decreases in tau). Hence, it does seem like the slowest group of participants could indeed be doing something extra across all orientations in order to provide "No" responses to reflected stimuli. Any specific conclusions in this regard, however, are precluded by the fact that neither the three-way Group $\times$ Stimulus Type $\times$ Orientation, $F(6,108)=0.428, p$ $>.70, M S E=51943$, nor the four-way Group $\times$ Stimulus Type $\times$ Orientation $\times$ Blocks, $F(6,108)=0.755, p>.50, M S E=32172$, interaction were significant in the mean reaction time analysis.

Faster and Slower Participants. In general, there are not too many (if any) mental rotation studies that have considered how responding might differ across faster and slower groups of participants within a study. Although it is tempting on the basis of the mean reaction time results obtained in this present study to conclude that processing is dramatically different for each type of participant (given that much steeper rotation functions and a greater separation of correct reaction times for the standard and reflected stimuli occurred for the slower group), it is actually the case that the mental rotation 
functions for the ex-Gaussian parameter mu are quite similar for both groups for the standard stimuli across orientations of 0 - 150 degrees (i.e., the corresponding slopes for the first five blocks are 1.182 and $1.189 \mathrm{~ms} / \mathrm{deg}$ for the fastest and slowest groups, respectively, and for the last five blocks they are 1.198 and $1.223 \mathrm{~ms} / \mathrm{deg}$, respectively; this equivalency can also be discerned by physically overlaying Figures $1 \mathrm{~A}$ and $1 \mathrm{~B}$ ). Hence, the rather more dramatic increase in mean reaction time with orientation for the slower group of subjects is due mainly to increases in the ex-Gaussian parameter tau. Such a result clearly indicates that the mental rotation process itself is generally quite analogous for both groups but is seemingly invoked in a very inefficient way by the slowest group of participants on a large number of trials.

Note as well though, that such individual differences in the ex-Gaussian parameter tau can also be regarded as a indicator that different types of processing strategies are being used by different individuals (Penner-Wilger, Leth-Steensen, \& Lefevre, 2002). For example, it could be the case that the slower participants are sometimes invoking a slower verbal-based processing strategy (like the two "deviant" participants in Bethell-Fox \& Shepard's, 1988, Experiment 2) or that they are sometimes using a less efficient analytical feature-by-feature rotation and comparison strategy instead of mentally rotating the stimuli holistically (Bethell-Fox \& Shepard, 1988; Petrusic, Varro, \& Jamieson, 1978; Rabbe et al., 2006). It could also be the case that the slower participants are double-checking the results of their rotation process more often than are the faster participants, which is consistent with the fact that the slower participants are also slightly more accurate. Although the timing of such a checking 
process should not then actually depend on orientation, perhaps it is the case that more checking is required as the angle of orientation of the stimuli increases.

Practice. In general, it is invariably found that the slope of the mental rotation function decreases with extensive practice (Bethell-Fox \& Shepard, 1988; Cohen \& Kubovy, 1993). One of the clearest demonstrations of this phenomenon was provided by Kail (1986) who found that the mental rotation slopes for eight 19- to 20-year old participants (on a two-stimulus mental rotation comparison task with letter and digit stimuli) decreased from about $2.5 \mathrm{~ms} / \mathrm{deg}$ in an initial session of 240 trials to about 0.7 $\mathrm{ms} / \mathrm{deg}$ by the $16^{\text {th }}$ session. Although a similar finding did indeed occur in the present study given that the steepness of the mental rotation functions for the mean correct reaction times were found to significantly decrease from the first five to the last five blocks of practice, interestingly, for both the faster and slower groups of participants, this effect was due to decreases in the slope of mental rotation functions for mu only over the two halves of the test session. Hence, for this study, reductions in slope with practice corresponded to shifts of the reaction time distributions (with greater shifts occurring for the higher orientations), suggesting two things (a) that the speed of mental rotation can be most directly tied to the values of mu and (ii) that the mental rotation process as a whole becomes more efficient with practice (i.e., it is not the case, in this study at least, that speed-ups in the rate of mental rotation are due to more efficient processing for the more slower set of responses in the tails of the reaction time distributions at the higher orientations).

One additional issue with respect to extensive practice is the degree to which participants might eventually begin to be able to circumvent the mental rotation process 
by memorizing the shapes of the stimuli at each of the various experienced orientations as the session progresses. Such a view can be characterized in terms of an instance-based memory retrieval process for which more and more instances of the letter shapes at each orientation, along with the responses made to them, become stored gradually allowing more and more of the subsequent responses to be made on the basis of direct retrieval of those instances rather than from mental rotation (Tarr \& Pinker, 1998).

One study which has examined this issue somewhat recently was undertaken by Heil, Rosler, Link, and Bajric (1998). These researchers had 37 participants perform a two-stimulus mental rotation comparison task across three sessions (separated by two weeks each) with 3-D Shepard and Metzler (1971) block figures as stimuli. The key manipulation in their study was that 16 of the participants also performed an additional four sessions of practice between the first and second test sessions. The practice stimuli involved a subset of 5 of the 15 standard stimuli used in the test sessions, rotated in only two of the three possible rotation axes (i.e., $x$ - or $y$-axis depth or $z$-axis plane rotations), at only a subset of the 20-degree incremented angular disparities (i.e., 20, 80, 100, and 160 degrees) used in the test sessions. For these participants, mean reaction times were indeed found to be much faster for stimulus conditions (i.e., same stimuli, same axis of rotation, at the same orientations) that were identical to those used in the practice sessions, although for some reason this effect also generalized somewhat to the nonpracticed orientations (i.e., 40, 60, 120 and 140 degrees). As well, Heil et al. (1998) found that both the overall drop in reaction times as well as the reductions in slope of the mental rotation functions across the three testing sessions were almost exactly the same for the control (i.e., no practice sessions) and experimental groups for test stimuli that 
were either not used in the added practice sessions or used but rotated along a different axis (i.e., no transfer to these stimulus conditions occurred). Heil et al. (1998) concluded that this latter finding was not consistent with process-based accounts for speed-ups in the rate of mental rotation with practice that assume either that practice enhances the general efficiency of the processes or subroutines involved in mental rotation process (Newell \& Rosenbloom, 1981) or that practice induces a change from a piece-by-piece to a holistic rotational strategy (Bethell-Fox \& Shepard, 1988).

With respect to the one-stimulus mental rotation task with 2-D letter stimuli that was used in the present study, the three accounts considered by Heil et al. (1998) would seem to make different predictions regarding the effect of practice on the slope of the mental rotation functions. Namely, process-based accounts would predict that although slopes should become shallower with practice they can never actually become flat. On the other hand, because the timing of a direct memory retrieval process should not depend on the angle of orientation, it would be expected under an instance-based account to find that that mental rotation slopes (for mean reaction times and also for both mu and tau) should eventually become flat. As mentioned, although the slopes of the mental rotation functions in the present study did decrease from the first five to the last five blocks of trials, they were certainly never flat.

However, one aspect of the present study was that three different letter stimuli were used whose presentations were intermixed throughout the blocks. Hence, it is possible that the present design did not provide the best conditions for the emergence of instance-based memory retrieval processing. To examine this possibility, this whole experiment was re-run with 18 new participants each of whom were presented with only 
one of the three letters throughout (i.e., "F", "L", or "P"), where otherwise every other aspect of the experiment was exactly the same as before. The reaction time results for this experiment are presented in Appendix B (note that 6 of these participants had to be dropped from this analysis because they had high error rates of $15 \%$ or more that were typically accompanied by a large number of either very slow or very fast reaction times below $100 \mathrm{~ms}$, both of which made fitting the ex-Gaussian quite difficult). Interestingly, the major aspect of this follow-up set of results is that they are very similar for the faster group to the results presented earlier (albeit about $100 \mathrm{~ms}$ faster overall) even though by the sixth block of test trials (which was actually the eighth block counting the two practice blocks) these participants would have responded to each standard and reflected version of the letter stimulus 21 times at each of the $30,60,90,120,150,210,240,270$, 300 and 330 degree orientations and 42 times at each of the 0 and 180 degree orientations. Such results seem to indicate quite clearly that although the use of mental rotation can indeed become very efficient, its usage cannot be completely eliminated.

\section{Conclusion}

In the present paper, the common finding that reaction time in the mental rotation task increases with angular departures of the test stimulus from upright was replicated. An ex-Gaussian analysis of the distribution of reaction times at each angle of orientation indicated that mean reaction time increases were accompanied by increases in the value of ex-Gaussian parameter mu. Importantly, because this parameter provides a measure of the locations of the leading edges (i.e., at the front end) of those distributions, this finding indicates that increasing the angle of orientation results in a shift of the reaction time distributions. Furthermore, both the nonlinearity that was found in the mental rotation 
functions and the changes in the slopes of those functions with practice were also reflected mainly in the ex-Gaussian parameter mu. The fact that most of the mental rotation phenomena observed here were accompanied by shifts of the reaction time distributions themselves is highly suggestive of the presence of a mental rotation process that is analogous to an actual physical rotation process, albeit one that can sometimes be circumvented (by at least some participants) if the test stimulus is close to being perceptually upright. Unfortunately though, these distributional findings themselves do not specifically rule out the possibility that some other (more analytical) process is being invoked in this task that could also account for the observed distributional shift effects.

Interestingly, the distributional analysis also revealed that differences in the slopes of the mental rotation functions between faster and slower participants were reflected mainly in the ex-Gaussian parameter tau. Because this parameter provides a measure of the size of the tail at the slow end of the distributions, this finding suggests that sets of reaction times obtained from the slower participants contain a larger proportion of very slow responses that is suggestive of the fact that they are sometimes using different strategies or performing double-checking operations. The fact that such individual differences in distribution of reaction times (and, hence, potentially in the underlying processing that is actually occurring in this task) is an important aspect to consider when interpreting the results of mental rotation studies in the future. 
Table 1

Significant Results Found in the ANOVA Analyses for Mean Correct Reaction Time

Main effects:

StimulusType, Blocks, Letters, Direction,

Orientation (linear and quadratic), Group

Two-way Interactions: $\quad$ StimulusType $\times$ Letters, StimulusType $\times$ Direction,

StimulusType $\times$ Orientation (linear and quadratic),

Blocks $\times$ Direction, Blocks $\times$ Orientation (linear),

Group $\times$ StimulusType, Group $\times$ Blocks,

Group $\times$ Orientation (linear)

Three-way Interactions: $\quad$ StimulusType $\times$ Letters $\times$ Direction,

StimulusType $\times$ Letters $\times$ Orientation,

Blocks $\times$ StimulusType $\times$ Direction 
Table 2

Mean Correct Reaction Time (in ms) as a Function of Angle of Orientation for Stimulus Type, the First and Last Five Blocks, and the Slowest and Fastest Group of Participants Angle of Orientation

\begin{tabular}{lllllll}
0 & 30 & 60 & 90 & 120 & 150 & 180 \\
\hline
\end{tabular}

Stimulus Type

$\begin{array}{lrrrrrrr}\text { Standard } & 656 & 680 & 735 & 804 & 887 & 997 & 1132 \\ \text { Reflected } & 777 & 826 & 876 & 953 & 1000 & 1071 & 1159\end{array}$

Blocks

$\begin{array}{lrrrrrrr}\text { First Five } & 750 & 792 & 838 & 932 & 994 & 1079 & 1215 \\ \text { Last Five } & 683 & 715 & 772 & 825 & 893 & 989 & 1075\end{array}$

Group

$\begin{array}{lrrrrrrr}\text { Fastest } & 609 & 623 & 638 & 686 & 727 & 794 & 888 \\ \text { Slowest } & 824 & 884 & 972 & 1070 & 1161 & 1274 & 1402\end{array}$

Mean

$\begin{array}{lllllll}716 & 753 & 805 & 878 & 944 & 1034 & 1145\end{array}$


Table 3

Proportion Correct as a Function of Angle of Orientation for Stimulus Type and Letters Angle of Orientation

\begin{tabular}{lllllll}
0 & 30 & 60 & 90 & 120 & 150 & 180 \\
\hline
\end{tabular}

Stimulus Type

$\begin{array}{llllllll}\text { Standard } & .993 & .996 & .989 & .986 & .966 & .904 & .830 \\ \text { Reflected } & .984 & .992 & .982 & .983 & .989 & .974 & .974\end{array}$

Letters

$\begin{array}{llllllll}\text { F } & .987 & .994 & .993 & .984 & .979 & .946 & .858 \\ \text { L } & .992 & .994 & .980 & .981 & .978 & .926 & .874 \\ \text { P } & .986 & .995 & .984 & .989 & .976 & .945 & .945\end{array}$

Mean

$\begin{array}{lllllll}.989 & .994 & .985 & .985 & .978 & .939 & .902\end{array}$


Table 4

Significant Results Found in the ANOVA Analyses for Mu, Sigma, and Tau $\underline{\mathbf{M u}}$

Main effects:

StimulusType, Blocks, Orientation (linear, quadratic, and cubic), Group

Two-way Interactions: $\quad$ Blocks $\times$ Orientation (linear and quadratic), Group $\times$ StimulusType, Group $\times$ Orientation (linear and quadratic)

\section{$\underline{\text { Sigma }}$}

Main effects:

StimulusType, Orientation (linear and quadratic), Group

Two-way Interactions: $\quad$ Group $\times$ Orientation (linear and quadratic) $\underline{\text { Tau }}$

Main effects: StimulusType, Blocks, Orientation (linear), Group

Two-way Interactions: $\quad$ Group $\times$ Orientation (linear and quadratic) 
Table 5

Mu (in ms) as a Function of Angle of Orientation for the First and Last Five Blocks and the Slowest and Fastest Group of Participants

Angle of Orientation

\begin{tabular}{lllllll}
0 & 30 & 60 & 90 & 120 & 150 & 180 \\
\hline
\end{tabular}

Blocks

$\begin{array}{llllllll}\text { First Five } & 538 & 557 & 583 & 617 & 664 & 722 & 884 \\ \text { Last Five } & 494 & 527 & 532 & 581 & 625 & 683 & 774\end{array}$

Group

$\begin{array}{llllllll}\text { Fastest } & 471 & 504 & 507 & 527 & 573 & 616 & 676 \\ \text { Slowest } & 561 & 581 & 608 & 670 & 716 & 790 & 982\end{array}$

Mean

$\begin{array}{lllllll}516 & 542 & 557 & 599 & 645 & 703 & 829\end{array}$


Table 6

Sigma and Tau (in ms) as a Function of Angle of Orientation for the Slowest and Fastest Group of Participants

\begin{tabular}{lllllll}
\multicolumn{8}{c}{ Angle of Orientation } \\
$0 \quad 30$ & 60 & 90 & 120 & 150 & 180 \\
\hline & Sigma
\end{tabular}

Group

$\begin{array}{llllllll}\text { Fastest } & 41 & 51 & 48 & 48 & 67 & 77 & 103 \\ \text { Slowest } & 63 & 52 & 71 & 87 & 113 & 118 & 207\end{array}$

Mean

$\begin{array}{lllllll}52 & 51 & 59 & 68 & 90 & 97 & 154 \\ & & \text { Tau } & & & \end{array}$

Group

$\begin{array}{llllllll}\text { Fastest } & 140 & 119 & 131 & 160 & 157 & 178 & 212 \\ \text { Slowest } & 266 & 303 & 370 & 407 & 450 & 492 & 425\end{array}$

Mean

$\begin{array}{lllllll}203 & 211 & 251 & 284 & 304 & 335 & 319\end{array}$


Table 7

Slope Values for the Mean Reaction Times and the Three Ex-Gaussian Parameters (in ms/deg) for Responses to Standard Stimuli for both the 0-90 and 90-180 Degree

Orientations for the Slowest and Fastest Group of Participants and the First Five and Last Five Blocks of Trials

\begin{tabular}{|c|c|c|c|c|c|c|c|c|}
\hline & \multicolumn{4}{|c|}{ Fastest Group } & \multicolumn{4}{|c|}{ Slower Group } \\
\hline & \multicolumn{2}{|l|}{$\underline{0-90}$} & \multicolumn{2}{|c|}{$90-180$} & \multicolumn{2}{|l|}{$\underline{0-90}$} & \multicolumn{2}{|c|}{$90-180$} \\
\hline & $\underline{1-5}$ & $\underline{6-10}$ & $\underline{1-5}$ & $\underline{6-10}$ & $\underline{1-5}$ & $\underline{6-10}$ & $\underline{1-5}$ & $\underline{6-10}$ \\
\hline Reaction Time & 0.793 & 0.810 & 3.197 & 2.560 & 2.717 & 2.323 & 4.697 & 4.117 \\
\hline $\mathrm{Mu}$ & 0.757 & 0.613 & 2.200 & 1.833 & 0.773 & 0.740 & 3.677 & 2.780 \\
\hline Sigma & 0.087 & 0.130 & 0.603 & 0.660 & 0.160 & 0.070 & 1.270 & 1.222 \\
\hline Tau & 0.010 & 0.183 & 0.850 & 0.883 & 2.017 & 1.667 & 0.970 & 1.347 \\
\hline
\end{tabular}




\section{Appendix 1}

The Quantile Maximum Likelihood Estimation (QMLE) Ex-Gaussian Fitting Procedure As advertised, QMLE fits the ex-Gaussian distribution to a set of quantile values that have been estimated from a set of reaction time data. Directly analogous to percentile values, quantiles represent the reaction time value for which a certain proportion of observed reaction times fall below it. For example, consider the following set of 10 ordered reaction times: $540,612,624,650,688,752,834,898,998,1112$. The $10^{\text {th }}$ quantile value represents the value for which exactly one-tenth of the reaction times fall below it. For this set of reaction times, that value represents the midpoint between the first two ordered values of 540 and 612 (i.e., 576), with the $20^{\text {th }}$ (i.e., 618), $30^{\text {th }}$ (i.e., 637), etc., quantiles being calculated accordingly (up to the $90^{\text {th }}$, resulting in nine possible quantiles in total; although note that if only four quantiles were required they would then correspond to the $20^{\text {th }}, 40^{\text {th }}, 60^{\text {th }}$, and $80^{\text {th }}$ quantile values).

Ex-Gaussian distributional curves are specified by the following formula

$$
f(t)=\left([1 / \tau] e^{\{-[(t-\mu) / \tau]+[\sigma 2 / 2 \tau 2]\}}\right)(\Phi\{[(t-\mu) / \sigma]-[\sigma / \tau]\}),
$$

where $t$ represents any particular reaction time value, $e$ is the exponential function, and $\Phi$ is the standard normal CDF (i.e., the last part of Equation A1 is equal to the probability under the normal curve below the z-score associated with the quantity within the braces after the $\Phi$ ). Different values of $\mu$ (i.e., mu), $\sigma$ (i.e., sigma), and $\tau$ (i.e., tau) yield a family of different ex-Gaussian curves with different shapes and different locations along the reaction time "X-axis". Because this curve has been specified mathematically, the area underneath it below any particular reaction time values can also be derived. Hence, given an ex-Gaussian curve specified by a particular set of $\mu, \sigma$, and $\tau$ values, quantiles values 
for that curve can be derived by determining the reaction time values for which, say, $10 \%$ of the area underneath the curve falls below it, for which $20 \%$ of the area underneath the curve falls below it, etc.

Essentially, what QMLE is doing is invoking a search for the values of $\mu, \sigma$, and $\tau$ that result in ex-Gaussian-based quantile values that most closely match the actual quantile values in a set of reaction time data. This search is undertaken using maximum likelihood techniques. More specifically, the best fitting ex-Gaussian curve is associated with the three ex-Gaussian parameter values for which the likelihood of the observed set of actual quantile values is a maximum. Note that for the set of 10 reaction times listed previously, the best fitting ex-Gaussian curve was the one for which the values of $\mu, \sigma$, and $\tau$ are 560,42 , and 197 , respectively. 
Appendix 2

Results for the Follow-up Study

Table A1

Significant Results Found in the ANOVA Analyses for Mean Correct Reaction Time in the Follow-up Study

Main effects:

StimulusType, Blocks, Orientation (linear and quadratic), Group

Two-way Interactions: $\quad$ Group $\times$ Orientation (linear)

Three-way Interactions: $\quad$ Blocks $\times$ StimulusType $\times$ Orientation (linear),

Group $\times$ StimulusType $\times$ Orientation (quadratic),

Group $\times$ Blocks $\times$ Direction, 
Table A2

Significant Results Found in the ANOVA Analyses for Mu, Sigma, and Tau in the Follow-up Study

$$
\underline{\mathrm{Mu}}
$$

Main effects:

StimulusType, Blocks, Orientation (linear), Group

Two-way Interactions: $\quad$ StimulusType $\times$ Orientation (linear)

Group $\times$ Orientation (linear)

\section{Sigma}

Main effects:

StimulusType, Blocks, Orientation (linear and cubic),

Group

Two-way Interactions: Group $\times$ Orientation (linear and cubic),

Blocks $\times$ Orientation (linear)

$\underline{\text { Tau }}$

Main effects:

Blocks, Orientation (linear), Group

Two-way Interactions: $\quad$ StimulusType $\times$ Orientation (linear) 
Table A3

Slope Values for the Mean Reaction Times and the Three Ex-Gaussian Parameters (in $\mathrm{ms} / \mathrm{deg}$ ) for Responses to Standard Stimuli for both the 0-90 and 90-180 Degree Orientations for the Slowest and Fastest Group of Participants and the First Five and Last Five Blocks of Trials in the Follow-up Study

\begin{tabular}{|c|c|c|c|c|c|c|c|c|}
\hline & Fastest & Group & & & & ower Gro & & \\
\hline & $\underline{0-90}$ & & $90-18$ & & $0-90$ & & $90-1$ & \\
\hline & $\underline{1-5}$ & $\underline{6-10}$ & $1-5$ & $\underline{6-10}$ & $1-5$ & $\underline{6-10}$ & $1-5$ & $\underline{6-10}$ \\
\hline Reaction Time & 1.460 & 1.333 & 2.823 & 2.310 & 2.913 & 1.750 & 4.417 & 3.387 \\
\hline $\mathrm{Mu}$ & 1.047 & 0.990 & 1.703 & 1.830 & 2.000 & 1.440 & 5.400 & 2.787 \\
\hline Sigma & 0.353 & 0.167 & 0.500 & 0.173 & 0.397 & -0.207 & 1.360 & 0.503 \\
\hline Tau & 0.407 & 0.373 & 1.130 & 0.517 & 0.867 & 0.440 & -0.507 & 0.560 \\
\hline
\end{tabular}




\section{References}

Bauer, B., \& Jolicoeur, P. (1996). Stimulus dimensionality effects in mental rotation. Journal of Experimental Psychology: Human Perception and Performance, 22, 82-94.

Bethell-Fox, C. E., \& Shepard, R. N. (1988). Mental rotation: Effects of stimulus complexity and familiarity. Journal of Experimental Psychology: Human Perception and Performance, 14, 12-23.

Brown, S., \& Heathcote, A. (2003). QMLE: Fast, robust, and efficient estimations of distribution functions based on quantiles. Behaviour Research Methods, Instruments, and Computers, 35, 485-492.

Brown, S., Cousineau, D., \& Heathcote, A. (2004). Technical manual for QMPE v2.18: Fortran code to fit response time distributions. Unpublished technical manual.

Cooper, L. A. (1975). Mental rotation of random two-dimensional shapes. Cognitive Psychology, 7, 20-43.

Cooper, L. A., \& Shepard, R. N. (1973). The time required to prepare for a rotated stimulus. Memory and Cognition, 1, 246-250.

Cohen, D., \& Kubovy, M. (1993). Mental rotation, mental representation, and flat slopes. Cognitive Psychology, 25, 315-382.

Eley, M. G. (1982). Identifying rotated letter-like symbols. Memory and Cognition, 10, 25-32.

Forster, B., Gebhardt, R.-P., Lindlar, K., Siemann, M., \& Delius, J. D. (1996). Mentalrotation effect: A function of elementary stimulus discriminability? Perception, 25, 1301-1316. 
Heathcote, A., Brown, S., \& Cousineau, D. (2004). QMPE: Estimating Lognormal, Wald, \& Weibull RT distributions with a parameter-dependent lower bound. Behavior Research Methods, Instruments, and Computers, 36, 277-290.

Heil, M., Rosler, F., Link, M., \& Bajric, J. (1998). What is improved if a mental rotation task is repeated - the efficiency of memory access, or the speed of a transformation routine. Psychological Research, 61, 99-106.

Hock, H. S., \& Tromley, C. L. (1978). Mental rotation and perceptual uprightness. Perception and Psychophysics, 24, 529-533.

Hockley, W. E. (1984). Analysis of response time distributions in the study of cognitive processes. Journal of Experimental Psychology: Learning, Memory and Cognition, 10, 598-615.

Kail, R. (1986). The impact of extended practice on rate of mental rotation Journal of Experimental Child Psychology, 42, 378-391.

Koriat, A., \& Norman, J. (1985). Mental rotation and visual familiarity. Perception and Psychophysics, 37, 429-439.

Newell, A., \& Rosenbloom, P. S. (1981). Mechanisms of skill acquisition and the law of practice. In J. R.. Anderson (Ed.), Cognitive skills and their acquisition (pp. 1-55). Hillsdale, NJ: Erlbaum.

Rock, I., \& Heimer, W. (1957). The effect of retinal and phenomenal orientation on the perception of form. American Journal of Psychology, 70, 493-511.

Penner-Wilger, M., Leth-Steensen, C., \& Lefevre, J.-A. (2002). Decomposing the problem size effect: A comparison of response time distributions across cultures. Memory \& Cognition, 30, 1160-1167. 
Petrusic, W. L., Varro, L., \& Jamieson, D. G. (1979). Mental rotation validation of two spatial ability tests. Psychological Research, 40, 139-148.

Pylyshyn, Z. W. (2002). Mental imagery: In search of a theory. Behavioral and Brain Sciences, 25, 157-238.

Raabe, S., Hoger, R., \& Delius, J. D. (2006). Sex differences in mental rotation strategy. Perceptual and Motor Skills, 103, 917-930.

Shepard, R. N., \& Metzler, J. (1971). Mental rotation of three-dimensional objects. Science, 171, 701-703.

Shepard, S., \& Metzler, D. (1988). Mental rotation: Effects of dimensionality of objects and type of task. Journal of Experimental Psychology: Human Perception and Performance, 14, 3-11.

Tarr, M. J., \& Pinker, S. (1989). Mental rotation and orientation-dependence in shape recognition. Cognitive Psychology, 21, 233-282.

Wiedenbauer, G., Schmid, J., \& Jansen-Osmann, P. (2007). Manual training of mental rotation. European Journal of Cognitive Psychology, 19, 17-36. 
Figure 1A

Fastest 10 Participants
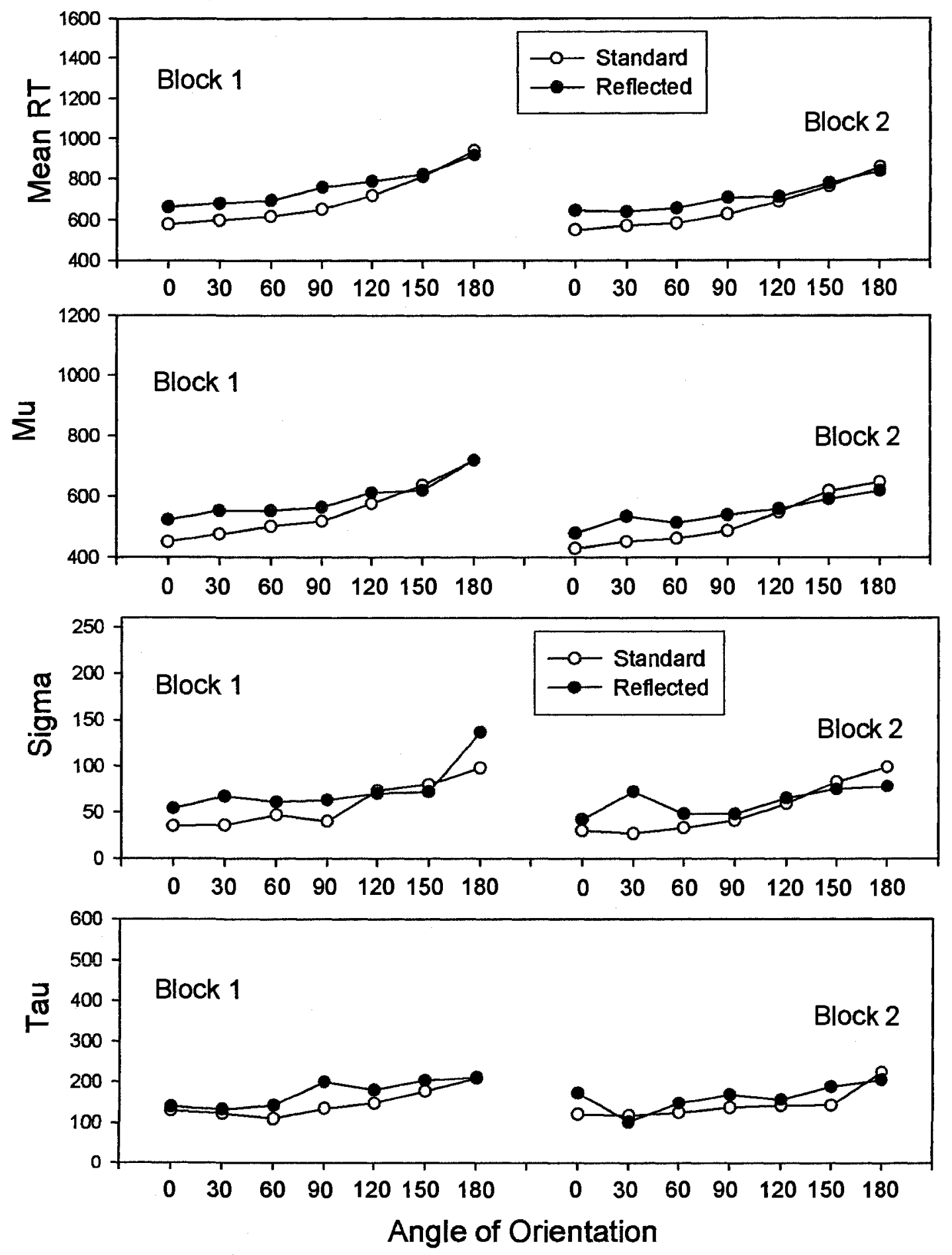
Figure 1B

\section{Slowest 10 Participants}
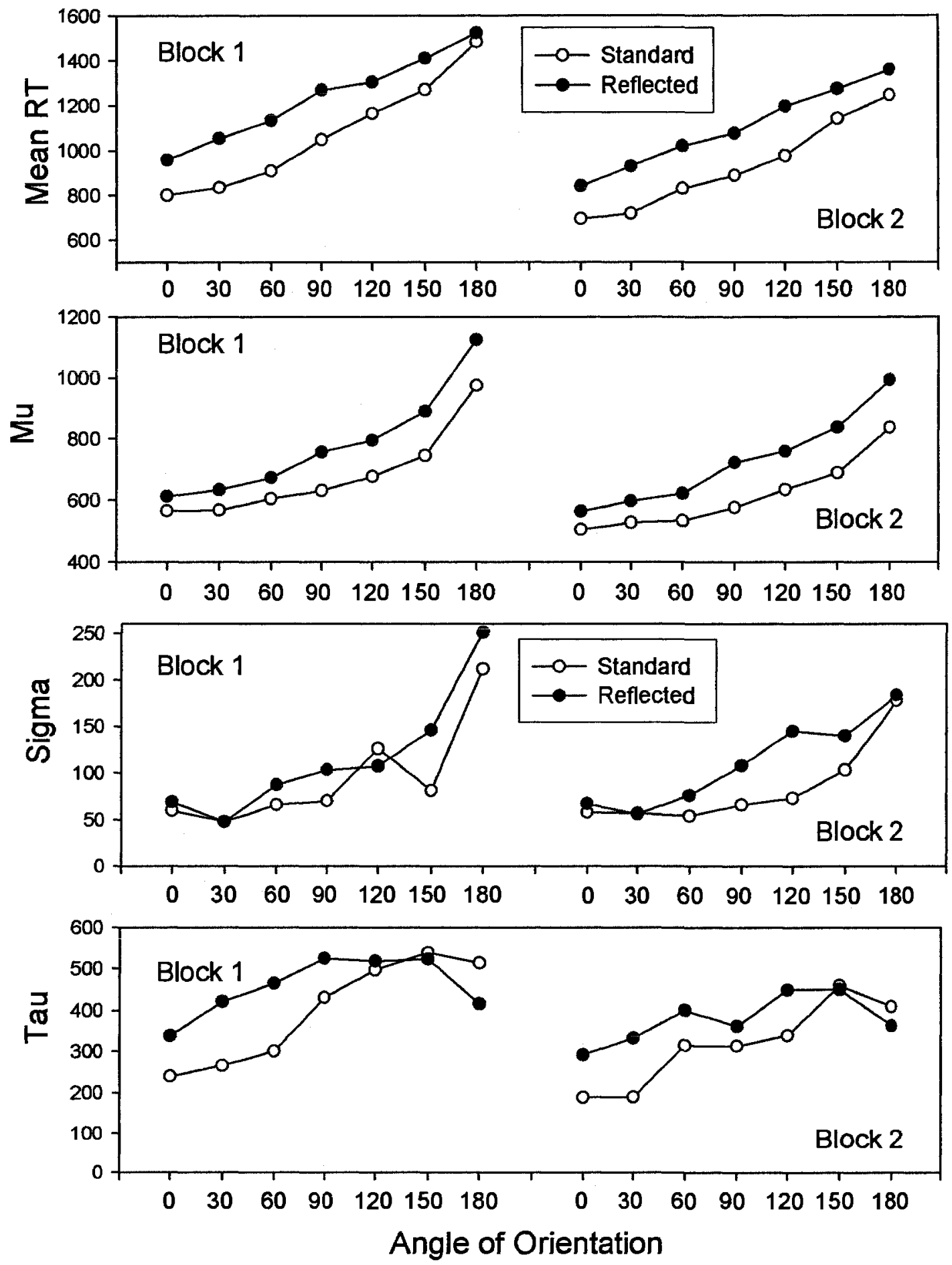
Figure 2A

\section{Fastest Participants}
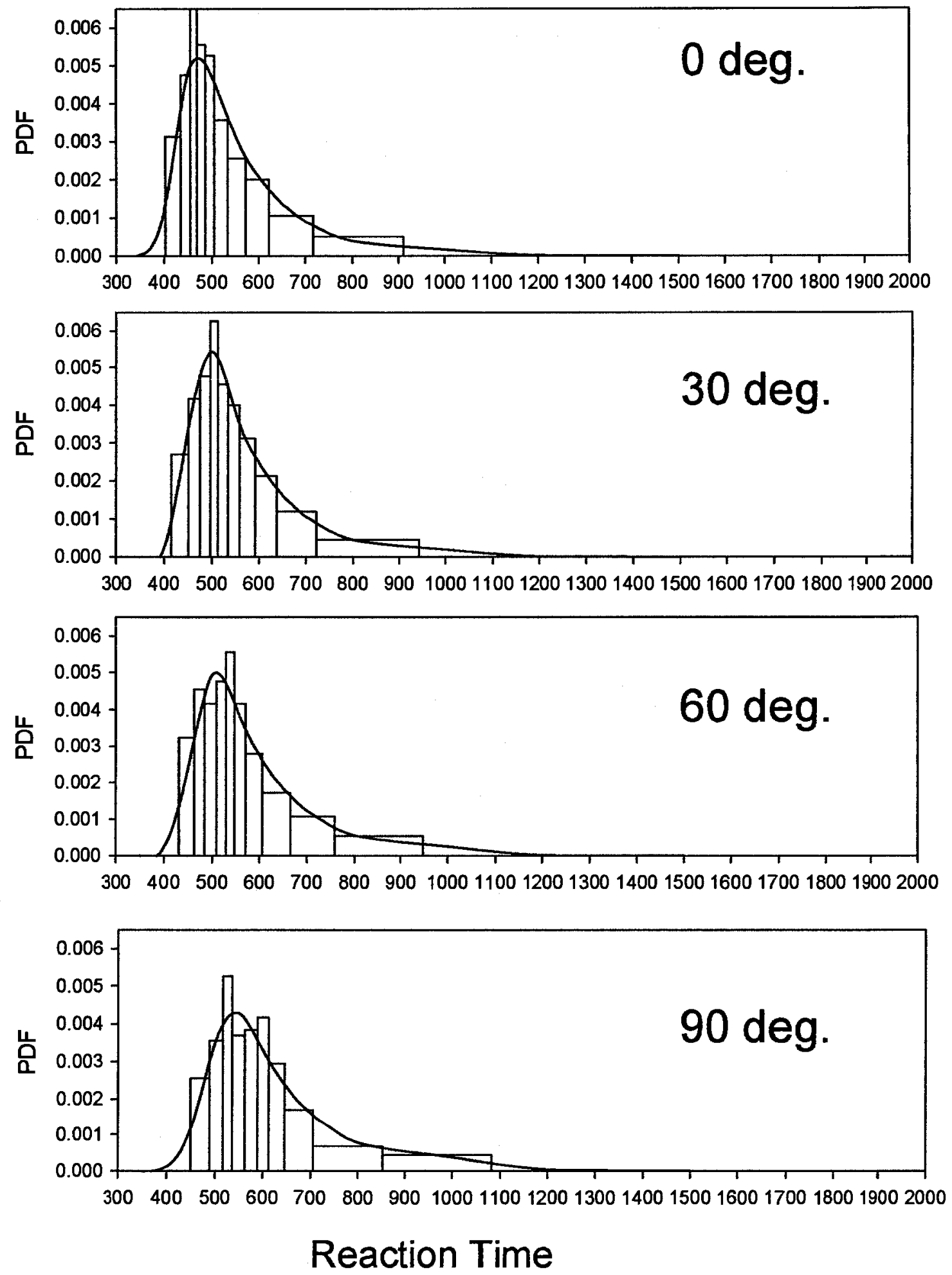
10 Fastest Participants
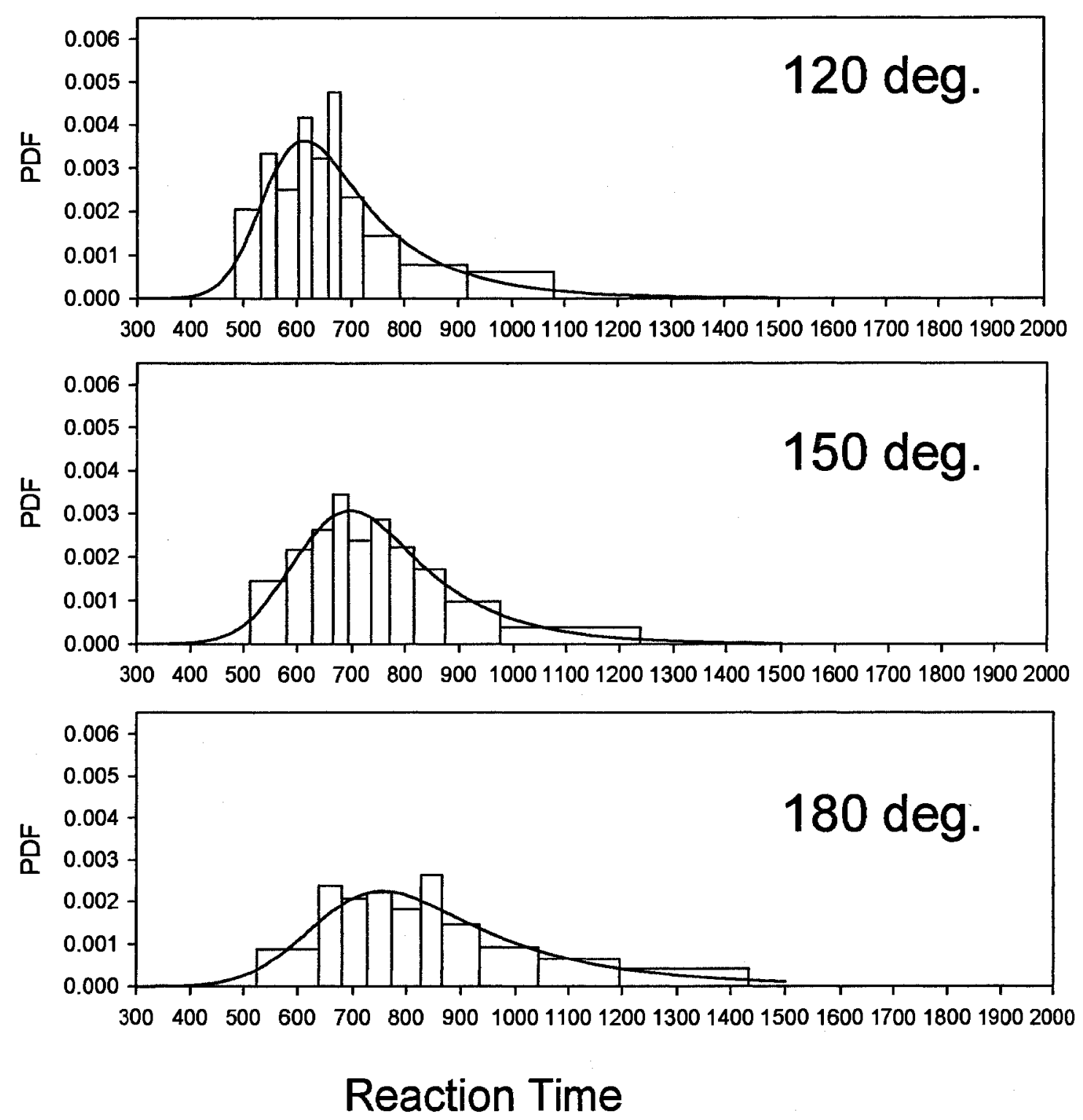
Figure 2B

\section{Slowest Participants}
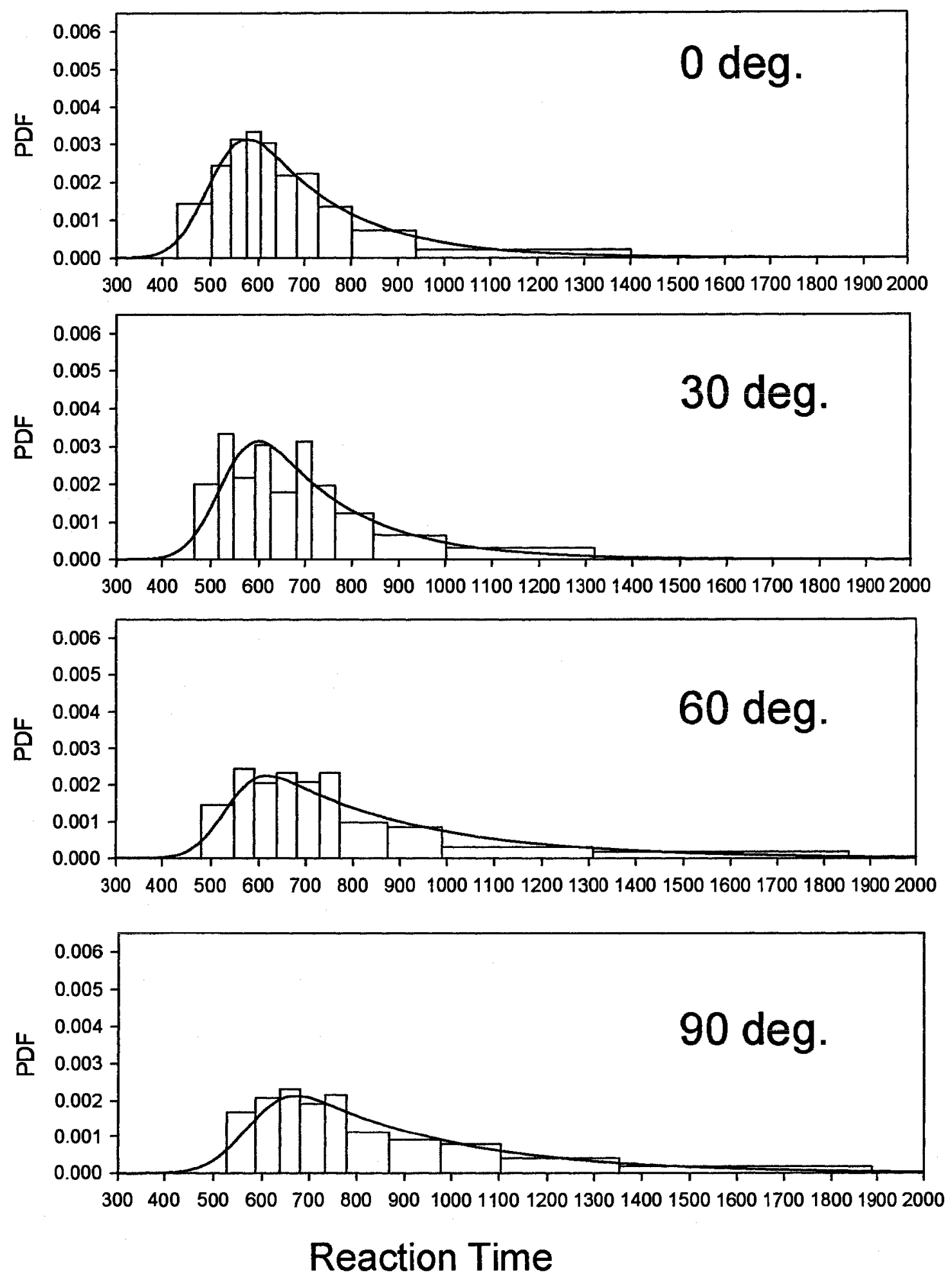


\section{Slowest Participants}
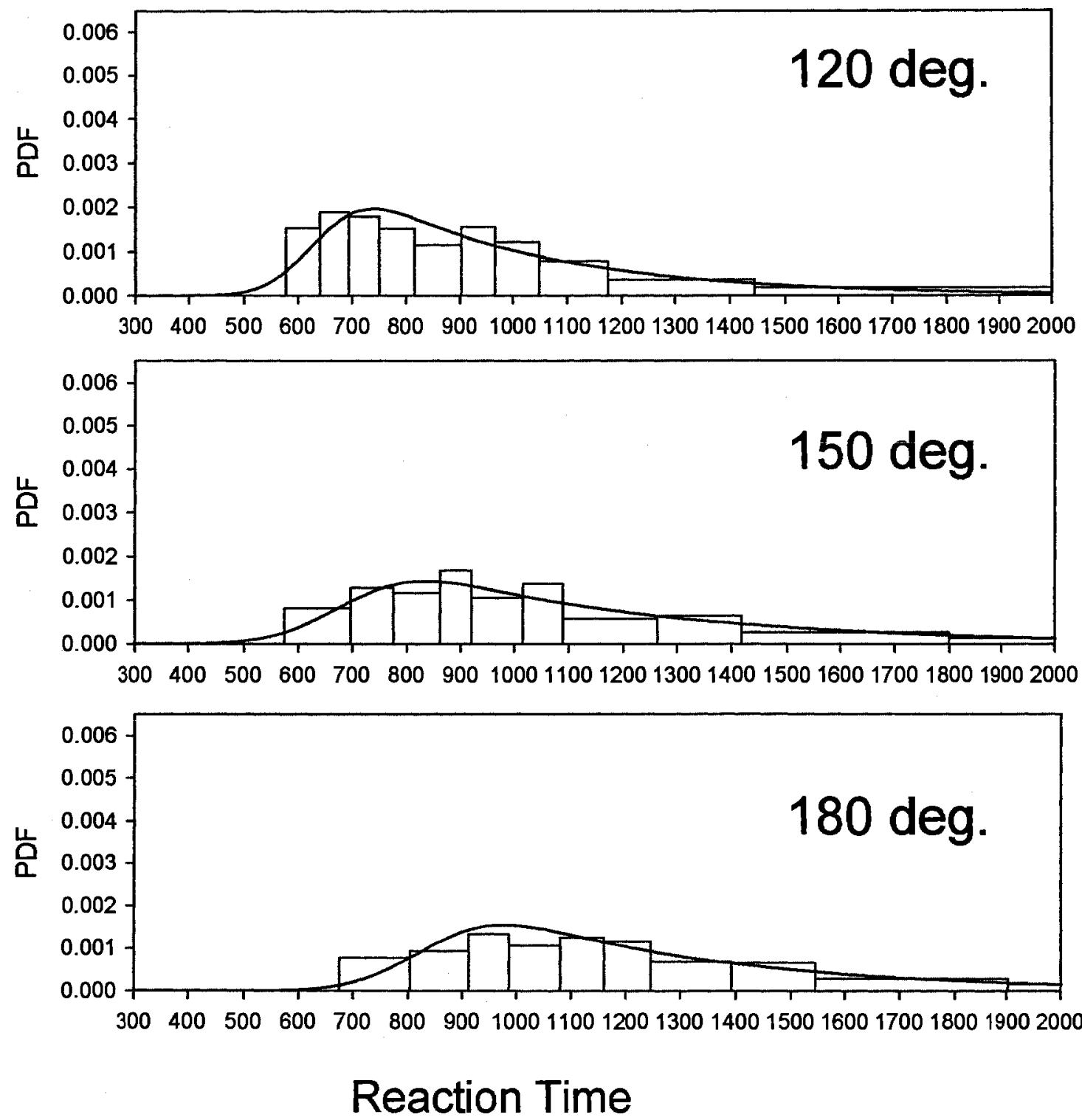
Figure A1

Fastest 9 Participants
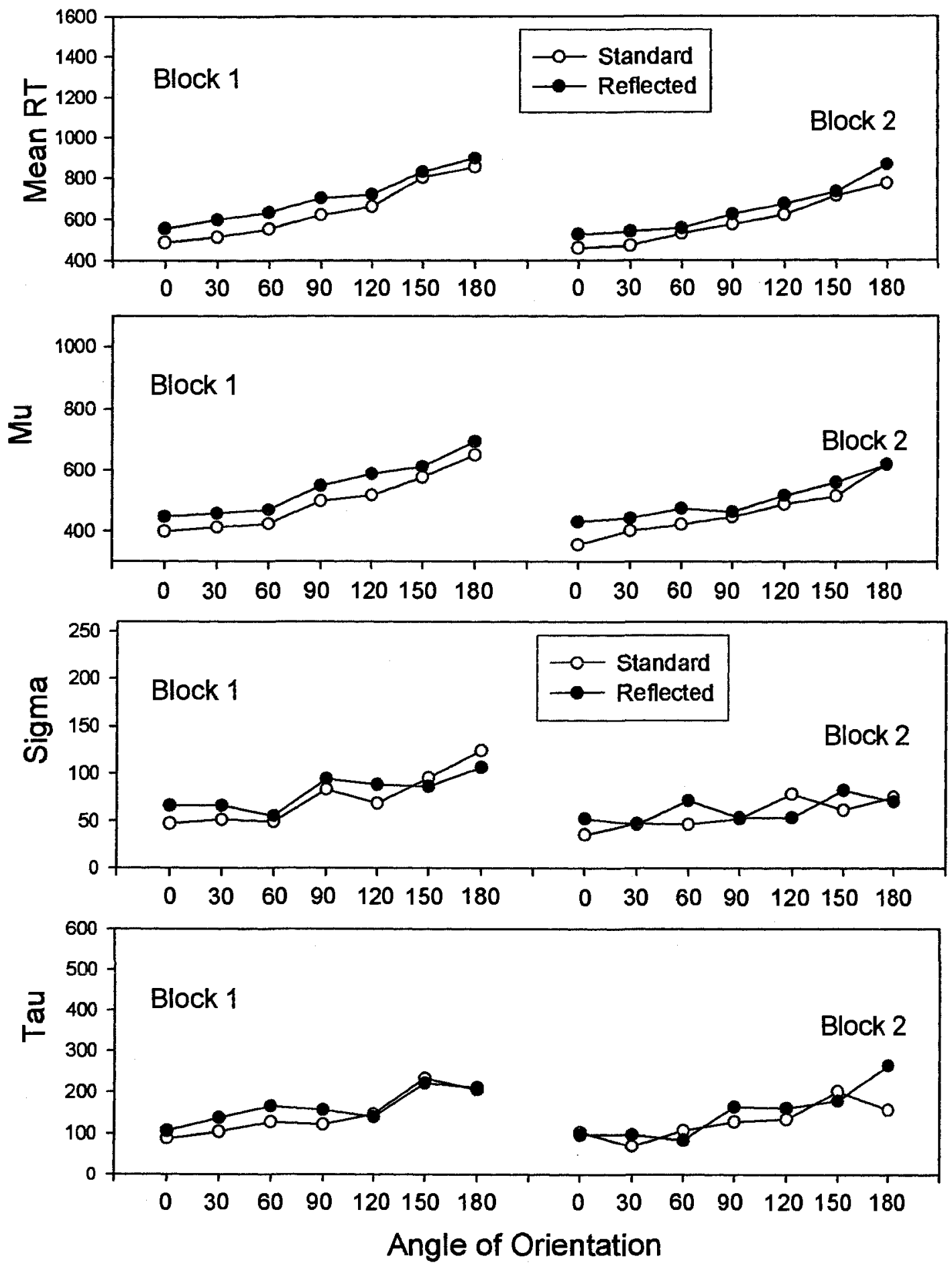
Figure A2

\section{Slowest 3 Participants}
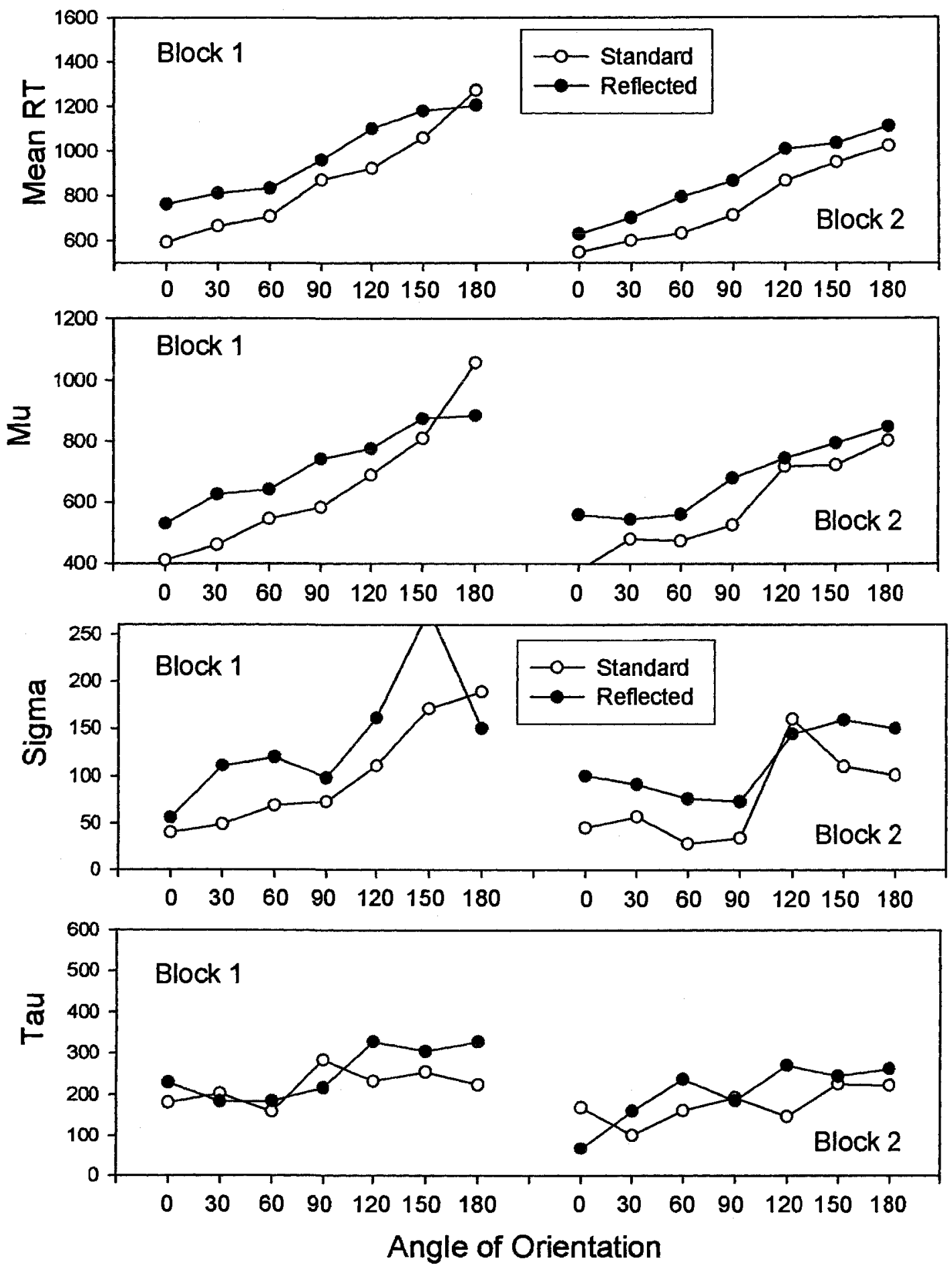